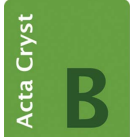

STRUCTURAL SCIENCE CRYSTAL ENGINEERING MATERIALS

ISSN 2052-5206

Received 2 March 2016

Accepted 25 May 2016

Edited by P. Bordet, Institut Néel, France

Keywords: bond lengths; coordination number; alkali metals; alkaline-earth metals.

Supporting information: this article has supporting information at journals.iucr.org/b

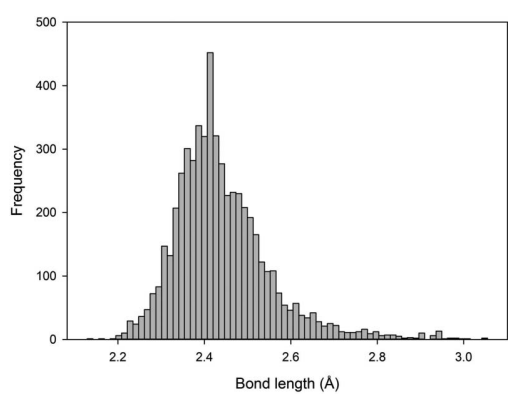

OPEN $\odot$ ACCESS

\section{Bond-length distributions for ions bonded to oxygen: alkali and alkaline-earth metals}

\author{
Olivier Charles Gagné* and Frank Christopher Hawthorne
}

Geological Sciences, University of Manitoba, 125 Dysart Road, Winnipeg, Manitoba R3T 2N2, Canada. *Correspondence e-mail: umgagneo@myumanitoba.ca

Bond-length distributions have been examined for 55 configurations of alkalimetal ions and 29 configurations of alkaline-earth-metal ions bonded to oxygen, for 4859 coordination polyhedra and 38594 bond distances (alkali metals), and for 3038 coordination polyhedra and 24487 bond distances (alkaline-earth metals). Bond lengths generally show a positively skewed Gaussian distribution that originates from the variation in Born repulsion and Coulomb attraction as a function of interatomic distance. The skewness and kurtosis of these distributions generally decrease with increasing coordination number of the central cation, a result of decreasing Born repulsion with increasing coordination number. We confirm the following minimum coordination numbers: ${ }^{[3]} \mathrm{Li}^{+},{ }^{[3]} \mathrm{Na}^{+},{ }^{[4]} \mathrm{K}^{+},{ }^{[4]} \mathrm{Rb}^{+},{ }^{[6]} \mathrm{Cs}^{+},{ }^{[3]} \mathrm{Be}^{2+},{ }^{[4]} \mathrm{Mg}^{2+},{ }^{[6]} \mathrm{Ca}^{2+},{ }^{26} \mathrm{Sr}^{2+}$ and ${ }^{[6]} \mathrm{Ba}^{2+}$, but note that some reported examples are the result of extensive dynamic and/or positional short-range disorder and are not ordered arrangements. Some distributions of bond lengths are distinctly multi-modal. This is commonly due to the occurrence of large numbers of structure refinements of a particular structure type in which a particular cation is always present, leading to an over-representation of a specific range of bond lengths. Outliers in the distributions of mean bond lengths are often associated with anomalous values of atomic displacement of the constituent cations and/or anions. For a sample of ${ }^{[6]} \mathrm{Na}^{+}$, the ratio $U_{\text {eq(Na) }} / U_{\text {eq(bonded }}$ anions) is partially correlated with $\left\langle{ }^{[6]} \mathrm{Na}^{+}-\mathrm{O}^{2-}\right\rangle\left(R^{2}=0.57\right)$, suggesting that the mean bond length is correlated with vibrational/displacement characteristics of the constituent ions for a fixed coordination number. Mean bond lengths also show a weak correlation with bond-length distortion from the mean value in general, although some coordination numbers show the widest variation in mean bond length for zero distortion, e.g. $\mathrm{Li}^{+}$in [4]- and [6]-coordination, $\mathrm{Na}^{+}$in [4]- and [6]-coordination. For alkali-metal and alkaline-earth-metal ions, there is a positive correlation between cation coordination number and the grand mean incident bond-valence sum at the central cation, the values varying from 0.84 v.u. for ${ }^{[5]} \mathrm{K}^{+}$to 1.06 v.u. for ${ }^{[8]} \mathrm{Li}^{+}$, and from 1.76 v.u. for ${ }^{[7]} \mathrm{Ba}^{2+}$ to 2.10 v.u. for ${ }^{[12]} \mathrm{Sr}^{2+}$. Bond-valence arguments suggest coordination numbers higher than [12] for $\mathrm{K}^{+}, \mathrm{Rb}^{+}, \mathrm{Cs}^{+}$and $\mathrm{Ba}^{2+}$.

\section{Introduction}

Many crystal structures have been refined in the past 100 years, and a large amount of information concerning interatomic distances in the solid state is available. There are many studies of bond-length distributions for specific pairs of ions, notably for cations bonded to oxygen [e.g. Baur, 1971 (Si); Burns et al., 1997 (U); Hawthorne et al., 2000 (S); Schindler et al., 2000 (V); Hawthorne \& Huminicki, 2002 (Be); Huminicki \& Hawthorne, 2002 (P); Mills \& Christy, 2013 (Te); Majzlan et al., 2014 (As)]. However, many of these studies have focused on subsets of the available information, both with regard to the number of ions and coordination numbers, and to the amount of data available for each example. We have examined the distribution of bond lengths for 135 ions bonded to oxygen 
in 462 configurations using 180331 bond lengths extracted from 9367 refined crystal structures; these data involve most ions of the periodic table and all coordination numbers in which they occur. Here we report the bond-length distributions for 10 ions, the common alkali-metal ions $\left(\mathrm{Li}^{+}, \mathrm{Na}^{+}, \mathrm{K}^{+}\right.$, $\mathrm{Rb}^{+}$and $\left.\mathrm{Cs}^{+}\right)$and alkaline-earth-metal ions $\left(\mathrm{Be}^{2+}, \mathrm{Mg}^{2+}, \mathrm{Ca}^{2+}\right.$, $\mathrm{Sr}^{2+}$ and $\left.\mathrm{Ba}^{2+}\right)$ in all observed coordination numbers where bonded to $\mathrm{O}^{2-}$ for a total of 63081 bond lengths in 7897 polyhedra from 4258 refined crystal structures. An advantage of working with a large number of ion pairs and a large amount of data is that it allows examination of subtle differences between the shapes of various distributions (e.g. bondlength distributions, mean-bond-length distributions) for various configurations of ions, which reflect differences in their bonding behaviour. These differences typically arise from either structural and/or electronic effects, and are well known for extreme examples such as [6]-coordinated $\mathrm{Cu}^{2+}$ and [6]-, [7]- and [8]-coordinated $\mathrm{U}^{6+}$; however, more subtle deviations from unimodality could be expected for the bond-length distributions of other ion configurations that are involved in related electronic or structural effects. Our motivation for this work is twofold: (1) The factors that affect bond distances are of continuing interest to all who work on crystal structures and their properties, and a comprehensive analysis of all the data should lead to increased understanding of those factors. Here we give a preliminary examination of the alkali-metal ions and alkaline-earth-metal ions in all observed coordination numbers where bonded to $\mathrm{O}^{2-}$, and make our complete dataset available for future more detailed work. (2) A comprehensive knowledge of the observed variation in bond lengths is critically important in assessing the validity of computational results on possible atomic arrangements (e.g. Richardson, 2013) and identifying unusual stereochemical features in newly solved or refined crystal structures.

\section{Definitions}

In the interest of clarity, we define certain terms that we use in the following text. We make no claims of generality; these are merely working definitions.

Chemical bond: There is no rigorous definition of a chemical bond that is useful in the context of the present work, which deals with some hundreds of thousands of observed interatomic distances. The decision on whether or not a specific interatomic distance corresponds to a chemical bond is made in terms of the local environment of the constituent atoms, e.g. is the distance consistent with a specific coordination number of the central ion, and is the valence-sum rule (Brown, 2002) reasonably well satisfied for the constituent ions? These are the criteria that are generally used for the listing of bond lengths in crystal-structure papers.

Coordination number: the number of counterions bonded to an ion.

Coordination polyhedron: The arrangement of counterions around an ion.

Ion configuration: A unique arrangement of ion type and coordination number.
Typical distribution: A distribution that is smooth and positively skewed, as for ${ }^{[6]} \mathrm{Na}^{+}$(Fig. 1).

\section{Coordination polyhedra}

3.1. Coordination polyhedra with the same coordination number

Most coordination numbers may show more than one coordination polyhedron. For example, [4]-coordination may be tetrahedral or square planar, [5]-coordination may be trigonal bipyramidal or square pyramidal, [6]-coordination may be octahedral or trigonal prismatic. However, within the bond-valence model (Brown, 2002), differences in angular arrangement of counterions have no effect on the valence sums, and hence we do not differentiate between different spatial configurations of coordination polyhedra with the same coordination number.

\subsection{The longest bond}

The determination of coordination number depends on how one defines a chemical bond (see 'definitions' section). It is fortunate that for many (if not most) crystal structures, there is a general consensus as to the coordination numbers observed. Thus rutile has $\mathrm{Ti}^{4+}$ in [6]-coordination and $\mathrm{O}^{2-}$ in [3]-coordination, and quartz has $\mathrm{Si}^{4+}$ in [4]-coordination and $\mathrm{O}^{2-}$ in [2]-coordination. For cations making a small number of bonds to their counterions, the determination of the coordination polyhedron is usually straightforward, and the coordination number can be assumed with confidence (e.g. for $\mathrm{Mg}^{2+}, \mathrm{Fe}^{2+}$, $\mathrm{Si}^{4+}$ ). However, making a decision on what is the longest bond for cations making a large number of bonds to their counterions is often less straightforward.

We examined the bond-length distributions for [3] + 1 (the latter being the fourth-shortest distance) and [4]-coordinated $\mathrm{Li}^{+}, \mathrm{Be}^{2+}, \mathrm{B}^{3+}, \mathrm{Na}^{+}$and $\mathrm{S}^{6+}$ to obtain a sense of the gap between the three shortest distances and the fourth distance

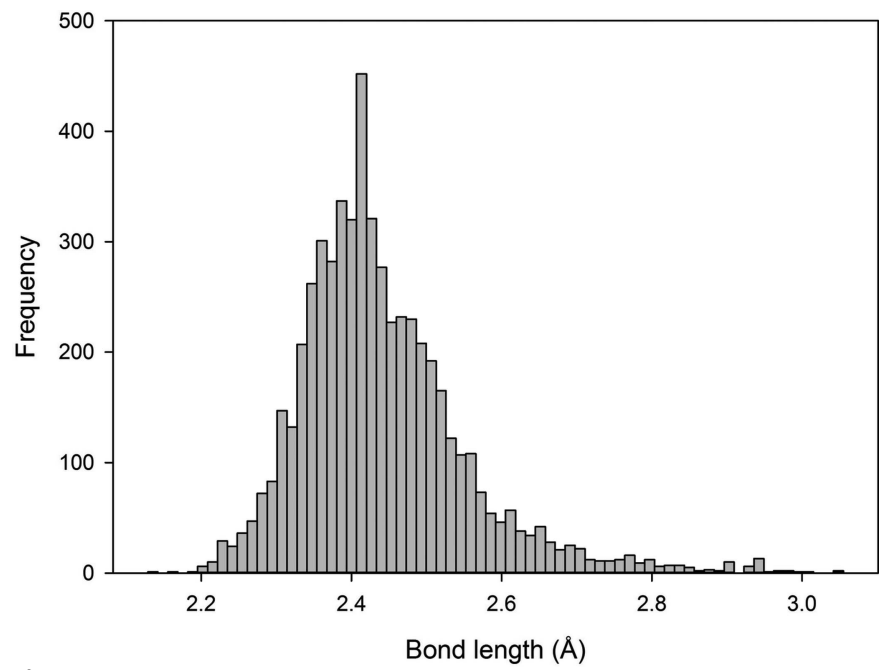

Figure 1

Bond length $(A)$

A typical distribution of bond lengths, shown for ${ }^{[6]} \mathrm{Na}^{+}$bonded to $\mathrm{O}^{2-}$ $(n=5532)$. 
for the coordination numbers [3] and [4]. Fig. 2(a) shows the ratio of the gap between the third- and fourth-shortest distances, and the mean bond length for the three shortest distances: $\left(l_{4}-l_{3}\right) /\left[\left(l_{1}+l_{2}+l_{3}\right) / 3\right]$ as a kernel density plot. ${ }^{1}$ For the coordination polyhedra we defined as [4], we observe a regular distribution with a mean value of 0.104 and minimum and maximum values of 0.000 and 0.333 , respectively. For $[3]+1$, the mean ratio is 1.056 and the minimum and maximum values are 0.198 and 2.521 , respectively. The distributions of the data are strikingly different, and for [3]and [4]-coordinated $\mathrm{Li}^{+}, \mathrm{Be}^{2+}, \mathrm{B}^{3+}, \mathrm{Na}^{+}$and $\mathrm{S}^{6+}$ suggest that the fourth-longest interatomic distance can be considered as bonded if $\left(l_{4}-l_{3}\right) /\left[\left(l_{1}+l_{2}+l_{3}\right) / 3\right]<0.333$ (Fig. 2a). We give analogous data for coordination numbers [8] +1 and [9] in Fig. $2(b)$ and [13] +1 and [14] in Fig. 2(c); in Fig. 2(b), we see a slight increase in the overlap between the distributions for [8] +1 and [9], a trend that continues for [13] + 1 and [14] in Fig. 2(c). Fig. 2 shows that, for higher coordination numbers, the determination of the longest bond is somewhat more ambiguous, but still fairly reliable in most cases.

\subsection{Large coordination numbers}

What large coordination numbers can we encounter in crystal structures? In terms of oxygen-based structures, Shannon (1976) lists ionic radii for coordination numbers up to [12], except for $\mathrm{Rb}^{+}$which he lists up to [14]. In different types of crystals, higher coordination numbers are common, particularly in Frank-Kasper phases. For example, A15 intermetallic alloys of the form $A_{3} B$ consist of [12]-coordinated $B$ atoms and [14]-coordinated $A$ atoms, and Laves $A B_{2}$ intermetallic phases involve [12]- and [16]-coordinated atoms. In terms of oxygen-based structures, it is probable that only the alkali-metal and alkaline-earth-metal ions may have coordination numbers exceeding [12], and we will pay special attention to structures in which such coordination numbers may be possible. For these cations, attempting to determine the coordination number from a list of nearest-neighbour anions is usually ambiguous. Some cations form ill-defined coordination polyhedra and require special attention. To this effect, we describe the procedure whereby we assign the coordination polyhedron of a cation below (\$4.2).

\section{Methods}

The DVD-ROM version of the ICSD with FindIt, Version 2010-2, was used for data collection for all ions bonded to oxygen. The collection of bond-length data was done on the basis of coordination polyhedra for all cations of the periodic table. A set of structures containing each ion pair of interest was accumulated for each cation. In these structure sets, only

\footnotetext{
${ }^{1}$ A kernel-density estimate shows the same distribution as a histogram. A histogram assigns an exact value to a piece of data, whereas a kernel-density estimate associates a variance with each piece of data and produces a much simpler plot. We use kernel-density estimates in this paper where we want to show several distributions on one figure, as several overlapping histograms are difficult to compare, whereas overlapping kernel-density estimates are visually more simple and much more easily compared.
}

the coordination polyhedra of the cation of interest were evaluated. The bonds in each coordination polyhedron were calculated and individually examined to ensure that only

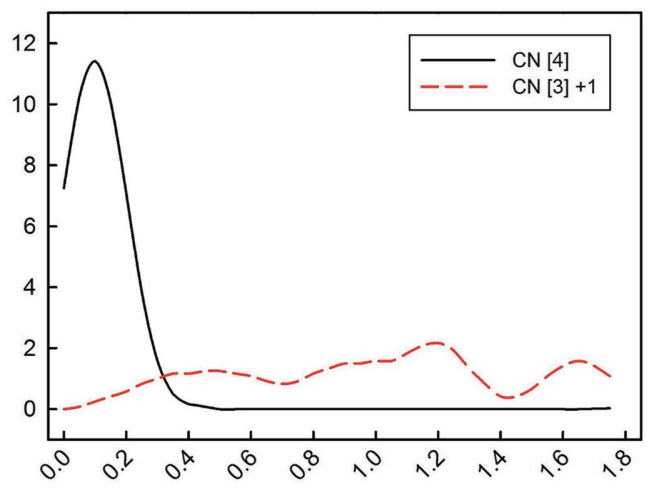

(a)

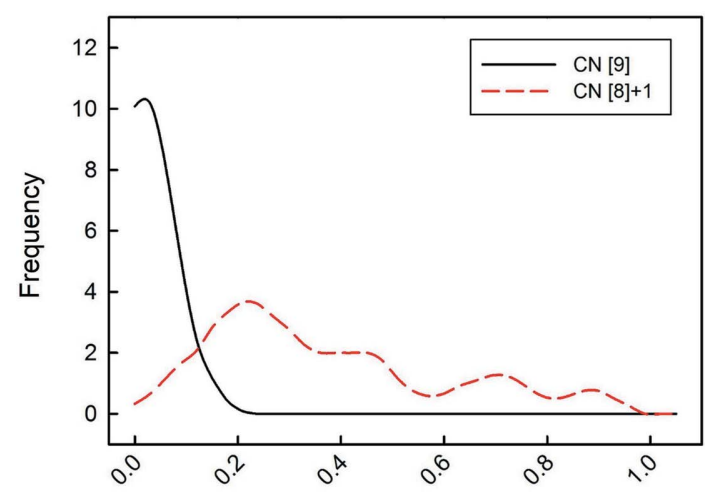

(b)

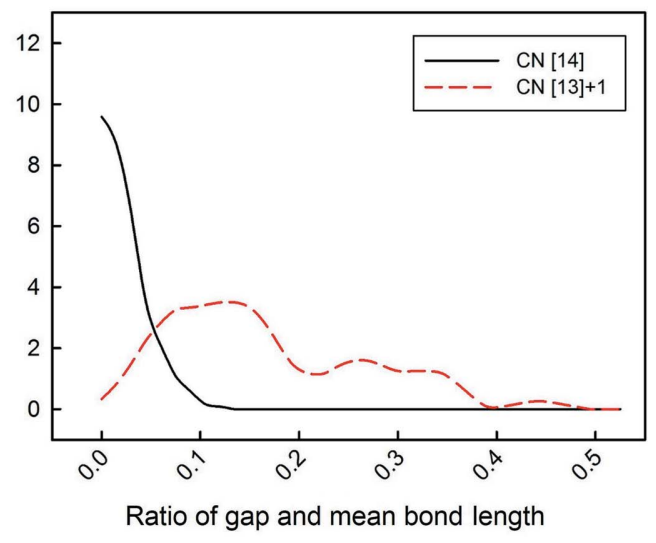

(c)

Figure 2

Ratio of the gap between the (a) third- and fourth-, $(b)$ eighth- and ninth-, $(c)$ thirteenth and fourteenth-shortest interatomic distance and the mean bond length of the $(a)$ three, $(b)$ eight, $(c)$ thirteen shortest interatomic distances for coordination numbers $(a)$ [3] and [4], $(b)$ [8] and [9] and $(c)$ [13] and [14]. Ions used are $(a) \mathrm{Li}^{+}, \mathrm{Be}^{2+}, \mathrm{B}^{3+}, \mathrm{Na}^{+}$and $\mathrm{S}^{6+} ;(b) \mathrm{Na}^{+}, \mathrm{Ca}^{2+}$, $\mathrm{Y}^{3+}, \mathrm{Te}^{4+}, \mathrm{La}^{3+} ;(c) \mathrm{K}^{+}, \mathrm{Rb}^{+}, \mathrm{Cs}^{+}, \mathrm{Ba}^{2+}$. The lack of overlap between the two distributions of Fig. 2(a) suggests that the fourth shortest distance for ions described as [3] is non-bonding. In Figs. 2(b) and (c), the increasing overlap between the two distributions shows that the determination of the exact coordination number for larger coordination polyhedra is more ambiguous. Sample sizes are $(a) n_{[3]+1}=41$ and $n_{[4]}=58$ coordination polyhedra; $(b) n_{[8]+1}=50$ and $n_{[9]}=50$ coordination polyhedra; $(c)$ $n_{[13]+1}=50$ and $n_{[14]}=47$ coordination polyhedra. 
suitable entries were included. Valid coordination polyhedra were not discarded due to problems elsewhere in the structure that have no effect on the coordination polyhedron of interest.

\subsection{Selection criteria}

The following criteria were used to select structure refinements:

(1) publication date $\geq 1975$;

(2) $R_{1} \leq 0.06$;

(3) the site of interest is fully occupied by the cation;

(4) all bonds involve ions at fully occupied sites;

(5) the cation and anion sites of interest show no positional disorder;

(6) crystallographic data were measured at ambient conditions;

(7) no data from powder, electron or synchrotron diffraction were included.

\subsection{Determination of coordination polyhedra}

The following guidelines were used to decide on the coordination polyhedron (and thus coordination number) of each cation treated. For most cations, the first few points were sufficient for a clear determination of the coordination number:

(1) The cation is bonded only to $\mathrm{O}^{2-}$.

(2) In general, we assumed that all cation-anion bonds are shorter than the shortest cation-cation distance for the coordination number of interest. ${ }^{2}$

(3) The ordered list of distances was examined for a hiatus in the increasing distances.

(4) We examined the effect of different cation-coordination numbers on the anion coordination.

(5) We compared the bond lengths with and without potential bonds to the data already gathered for the cation to see if the behaviour resembled that of one coordination number more than another.

(6) After $\sim 10 \%$ of the structures had been processed for a specific ion pair, we examined the files for different coordination numbers of the same cation for potential trends and inconsistencies.

(7) We examined the chemical formula for the presence of unrefined $\mathrm{H}$ atoms. This was mostly relevant in locating weak bonds between the cation of interest and the $\mathrm{O}$ atom of an $\left(\mathrm{H}_{2} \mathrm{O}\right)$ group. Where $\mathrm{H}$ atoms were not located in the refinement, and such bonds seemed plausible, the data were discarded.

(8) We plotted the structure to obtain a visual sense of any ambiguity.

\footnotetext{
${ }^{2}$ There are notable exceptions to this criterion. For example, in the structure of $\mathrm{RbLiO}$ (Sabrowsky \& Vogt, 1987), $\mathrm{Rb}^{+}$is surrounded by six $\mathrm{O}^{2-}$ ions with distances $2.897,2.927 \times 2,3.247 \times 2$ and $3.488 \AA$, but the shortest $\mathrm{Rb}-\mathrm{Li}$ distance is $2.916 \AA$, shorter than five of the six shortest $\mathrm{Rb}^{+}-\mathrm{O}^{2-}$ distances. Another example occurs in $\mathrm{RbNaO}$ (Sabrowsky, Thimm \& Vogt-Mertens, 1985 ); $\mathrm{Rb}^{+}$is surrounded by six $\mathrm{O}^{2-}$ ions with distances $2.905,3.066 \times 4$ and $3.589 \AA$, but the shortest $\mathrm{Rb}-\mathrm{Na}$ distance is $3.089 \AA \times 4$, very similar to the four intermediate length $\mathrm{Rb}^{+}-\mathrm{O}^{2-}$ distances. Similar behaviour is shown by $\mathrm{KNaO}$ (Sabrowsky \& Schroeer, 1982). However, this behaviour appears to be confined to mixed alkali-metal oxides.
}

(9) Very sparingly, we used bond-valence curves to determine whether the inclusion or omission of bonds gave better bond-valence sums. However, little weight was given to this method because (1) it is circular, and (2) some published bond-valence parameters were doubtful at the time of this evaluation.

We plotted the bond-length frequency distributions for all ion configurations to identify obvious outliers that originated from (1) gross errors in the refinement, (2) errors in the ICSD entry, and/or (3) collection errors on our part. Arbitrary (often dynamic) limits were set for the lower and upper tails of the distribution of each ion configuration, whereby every entry with at least one bond below the former or above the latter was either (1) verified and confirmed in the original publication, or (2) discarded as an error. In particular, minerals may show considerable chemical zoning within individual grains and also significant grain-to-grain compositional differences, depending on the details of paragenesis. Where this is the case, there may be significant differences between the actual composition of the crystal and that assumed for the crystal used to collect the X-ray intensity data. Thus, errors regarding site occupancy may occur (unless the composition of that grain is analyzed by electron- and ion-microprobe), leading to apparently anomalous bond lengths. For entries with abundant data (e.g. $\mathrm{Si}^{4+}$ with over 10500 bond distances), the entries were verified in terms of increasing bond lengths for the lower cut-off, and decreasing bond lengths for the upper cut-off, until a reliable series of entries were confirmed with those bond lengths. For configurations with very little data $(\sim 3$ or less coordination polyhedra), all entries were verified.

Taking into account the large amount of crystal structures examined, it was generally safer to discard a doubtful entry than it was useful to have its bond-length information included with reliable data, except where there was a paucity of bond lengths for the cation and coordination number(s) of interest.

Gagné \& Hawthorne (2015) found that the agreement with the valence-sum rule of Brown (2002) is better when including the longer bonds in higher coordination polyhedra. As we shall see later, this is the case for the alkali and alkaline-earth metals, and also for configurations involving lone-pairstereoactive cations (manuscript in preparation). Thus, long bonds obtained from the collection procedure described above were included.

This procedure resulted in 55 configurations involving 4859 coordination polyhedra and 38594 individual bond lengths for the alkali metals, and 29 configurations involving 3038 coordination polyhedra and 24487 individual bond lengths for the alkaline-earth metals. We make our complete dataset available for future more detailed work.

\section{Shape of the bond-length distributions}

Bond-length distributions commonly resemble a positively skewed Gaussian distribution. The shape originates from the variation in Born repulsion and Coulomb attraction as a function of interatomic distance. Two useful statistical measures used to describe the shape of these distribution are 
skewness and kurtosis. Skewness is a measure of the asymmetry of the distribution about its mean, and can be positive (as in Fig. 1 for ${ }^{[6]} \mathrm{Na}^{+}$) or negative. Kurtosis is a measure of the distribution of data between the peak and the tails of the distribution: a high kurtosis indicates that the distribution has a sharper maximum and larger tails, and a low kurtosis indicates that the distribution has a rounder maximum and smaller tails. Thus, important data that we derive from the bondlength distributions determined here are mean bond length, skewness and kurtosis. Deviations from this typical shape are frequent, and can be the result of structural and/or electronic effects that result in emergent bond-length constraints. Hence, we can gain insight into the reasons underlying the bonding behaviour of atoms from a visual inspection of their bondlength distributions, e.g. the familiar $(4+2)$ bimodal distribution of bond lengths for octahedrally coordinated $\mathrm{Cu}^{2+}$ from the Jahn-Teller effect (Jahn \& Teller, 1937), associated with the degenerate electronic ground state of a $d^{9}$ metal in a holosymmetric octahedral field.

\section{Effect of sampling}

\subsection{Sample size}

A critical issue involved in the calculation of mean bond length, skewness and kurtosis is whether the sample size (number of bond lengths) is sufficiently large to ensure a representative distribution. We examined this issue using the data of Fig. 1, calculating the mean bond length, skewness and kurtosis for many different sample sizes and examining the values as a function of sample size compared with the values for the parent distribution (mean bond length $=2.441$ with a standard deviation of $0.112 \AA$; skewness $=1.32$, kurtosis = 3.25). The results are shown in Fig. 3. In the range 5500-1000 bonds, the values of mean bond length (Fig. $3 a$ ) and its standard deviation (Fig. 3b), skewness (Fig. 3c) and kurtosis (Fig. $3 d$ ) varied between 2.437-2.444 and 0.109-0.119 А, 1.23-1.51 and $2.75-4.08$, respectively; in the range $1000-100$, the values of mean bond length and its standard deviation, skewness and kurtosis varied between 2.433-2.446 and 0.104-0.122, 1.021.73 and 1.60-5.28, respectively; below 100 , the values of skewness and kurtosis varied from 2.424-2.471 and 0.078$0.159 \AA$, from -1.24 to 3.11 and from -1.49 to 11.72 , respectively. Similar results were obtained (scaled by a factor of 6) by using polyhedra rather than individual bond lengths. As a result of these large variations, we do not list skewness and kurtosis values for sample sizes of less than 100 bonds in this work, and we note that the values for sample sizes up to 1000 bonds may be associated with non-negligible error. We do list mean bond lengths as this is important information for ongoing work on these materials, but we emphasize that the values listed may be adversely affected by small sample size.

\subsection{The effect of outliers}

Values of skewness and kurtosis are very sensitive to the presence of outliers. For example, our dataset for ${ }^{[7]} \mathrm{Cs}^{+}$(Fig. S1aq of the supporting information) contains two coordina- tion polyhedra with bonds longer than $3.75 \AA$, while the longest bond for the eight other polyhedra varies in the range 3.3-3.5 ̊. Calculating the skewness and kurtosis with and without the two polyhedra with bonds longer than $3.75 \AA$, we obtain values of skewness and kurtosis of 1.51 and 3.04 (with), and 0.62 and -0.31 (without), respectively. Careful evaluation of the structure and of the probable longest bond (see \$3.2) has greatly reduced the adverse effect of outliers on skewness and kurtosis in our analyses, but one must be careful of this

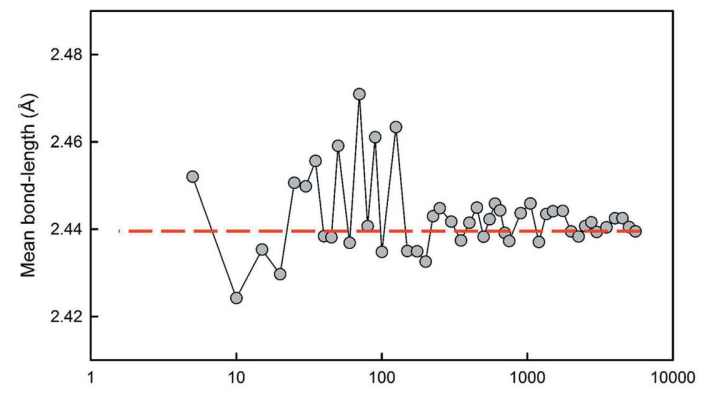

(a)

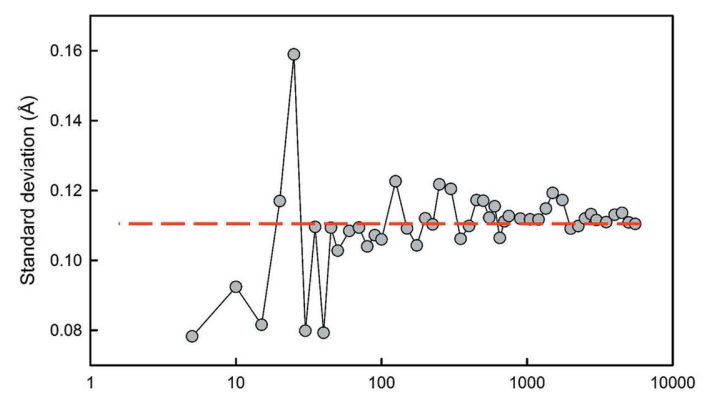

(b)

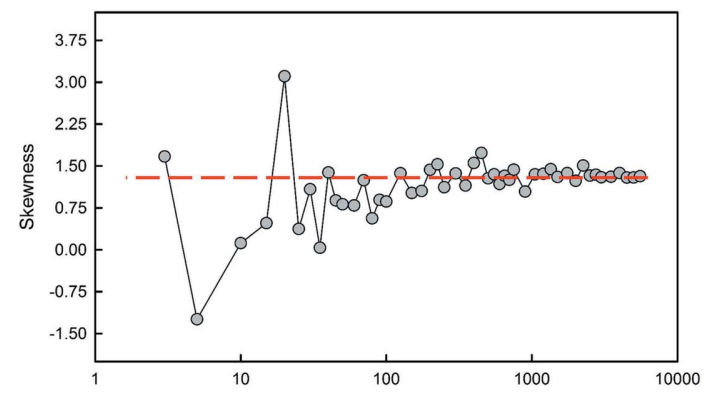

(c)

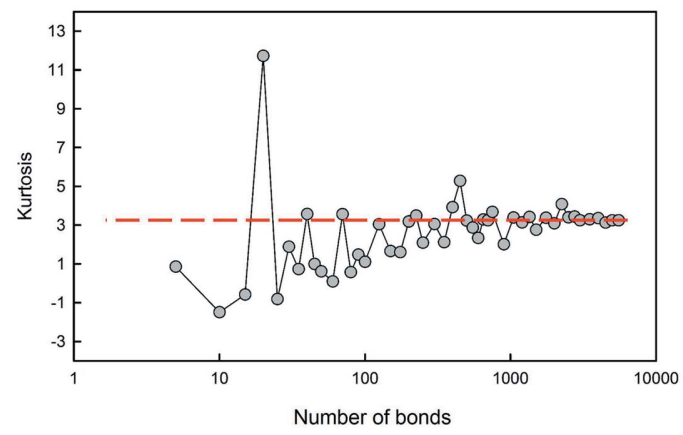

(d)

Figure 3

The effect of sample size on $(a)$ mean bond length, $(b)$ standard deviation of the mean bond length, $(c)$ skewness and $(d)$ kurtosis. The dashed line shows the value for the parent distribution. 
issue as a single errant data point for a confirmed structure can change the values considerably.

\subsection{Non-random sampling}

Another issue that can affect skewness and kurtosis is the occurrence of spikes in the distribution of bond lengths due to extensive work on specific structure types. A prominent example is shown in Fig. 4: the bond-length distribution for ${ }^{\left[{ }^{6]}\right.} \mathrm{Cr}^{3+}$. There is a fairly typical distribution except for a prominent spike at $1.99 \AA ̊$ A where $\sim 70$ distances lie above the trend of the general distribution. Examination of the data shows that these distances originate from Lenaz et al. (2004); these authors refined structures from the solid solution $\left(\mathrm{Mg}, \mathrm{Fe}^{2+}\right) \mathrm{Cr}_{2}^{3+} \mathrm{O}_{4}$ with the spinel structure, and these contributed 11 structures in which the $\mathrm{Cr}^{3+}-\mathrm{O}^{2-}$ distances are symmetry-constrained to be identical in each structure, providing 66 distances of $\sim 1.99 \AA$ and accounting for the spike in the distribution of Fig. 4.

Thus, §\$6.1-6.3 emphasize that the numerical values for skewness and kurtosis must be interpreted with care. While the presence of trends in skewness and kurtosis gives us structural information, the absence of such trends may be due to sampling issues.

\section{Results for the alkali metals}

Our collection and filtering criteria resulted in a combined sample size of 38594 bonds and 4859 coordination polyhedra. Table 1 gives the 55 observed configurations, the mean bond length and standard deviation, the minimum and maximum bond length (and range), the skewness and kurtosis (where justified by sample size), and the number of bonds and coordination polyhedra for the five common alkali-metal ions. Fig. S1 gives all the bond-length distributions for the alkali metals; those with adequate sample sizes (as discussed above) are

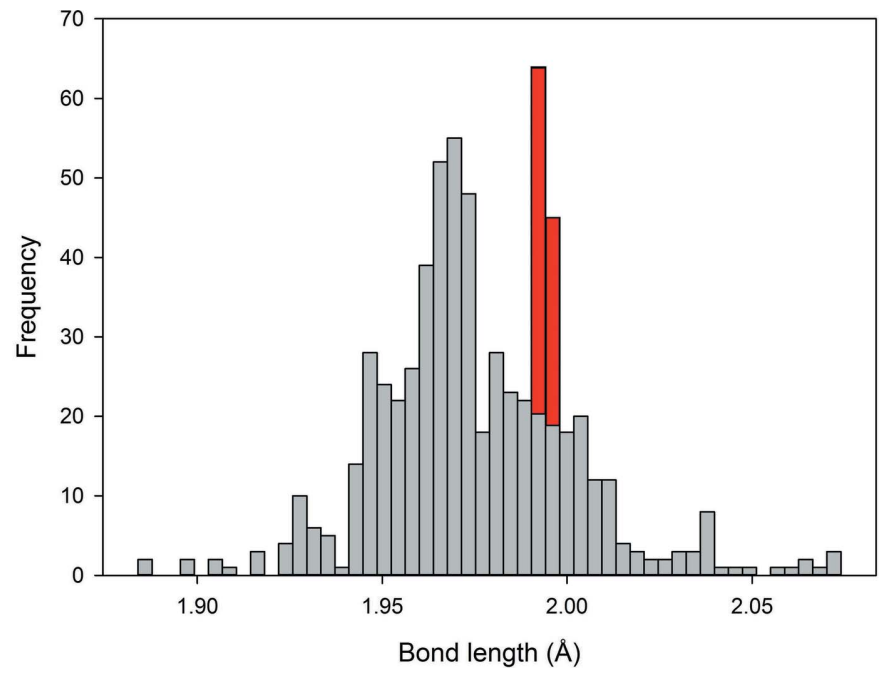

Figure 4

Bond-length distribution for ${ }^{[6]} \mathrm{Cr}^{3+}$ bonded to $\mathrm{O}^{2-}(n=624)$. A spike of data is observed at $1.99 \AA$ due to extensive work done on the spinel structure. shown in Fig. 5. An important issue is the reliability of the data at the limits of its distribution, i.e. at the lowest and highest observed coordination numbers for each ion, and below we examine the data at the lower and upper limits of these distributions.

\subsection{Observed coordination numbers}

Fig. 6 shows the variation of the coordination numbers for the alkali-metal ions. The minimum coordination number increases from [3] for $\mathrm{Li}^{+}$and $\mathrm{Na}^{+}$to [4] for $\mathrm{K}^{+}$and $\mathrm{Rb}^{+}$to [6] for $\mathrm{Cs}^{+}$, and there is a corresponding increase in the maximum coordination number from [8] to [20]. The number of coordination numbers also increases along the series, from 6 for $\mathrm{Li}^{+}$to 15 for $\mathrm{Rb}^{+}$and $\mathrm{Cs}^{+}$.

7.1.1. $\mathrm{Li}^{+} . \mathrm{Li}^{+}$has six coordination numbers from [3] to [8], with a strong preference for coordination number [4] $(n=419$ coordination polyhedra). ${ }^{[3]} \mathrm{Li}^{+}$is observed in only four coordination polyhedra in four structures: $\mathrm{Li}_{2} \mathrm{Yb}_{5} \mathrm{O}_{4}\left(\mathrm{BO}_{3}\right)_{3}$ (Jubera et al., 2001); KLiO (Sabrowsky, Mertens \& Thimm, 1985); RbLiO (Sabrowsky \& Vogt, 1987); $\mathrm{LiBa}\left(\mathrm{B}_{9} \mathrm{O}_{15}\right)$ (Pushcharovskii et al., 2002). The observed mean bond lengths are $1.958,1.891,1.915$ and $1.888 \AA$, respectively, and the incident bond-valence sums (using the parameters of Gagné \& Hawthorne, 2015) are $0.744,0.825,0.797$ and 0.829 v.u., respectively. The displacement parameters for ${ }^{[3]} \mathrm{Li}^{+}$in $\mathrm{Li}_{2} \mathrm{Yb}_{5} \mathrm{O}_{4}\left(\mathrm{BO}_{3}\right)_{3}$ are an order of magnitude larger than those for $\mathrm{Yb}^{3+}$ and twice that of ${ }^{[4]} \mathrm{Li}^{+}$. Similarly, in $\mathrm{LiBa}\left(\mathrm{B}_{9} \mathrm{O}_{15}\right), U_{\text {eq }}$ for ${ }^{[3]} \mathrm{Li}^{+}$is $\sim 8$ times $U_{\text {eq }}$ for both the ${ }^{[3]} \mathrm{B}^{3+}$ and ${ }^{[4]} \mathrm{B}^{3+}$ cations (and the $\mathrm{O}^{2-}$ anions). These results suggest significant (dynamic or static) positional disorder. However, in KLiO, $U_{\text {eq }}$ for ${ }^{[3]} \mathrm{Li}^{+}$is similar to the $U_{\text {eq }}$ values for $\mathrm{K}^{+}$and $\mathrm{O}^{2-}$, and there is no reason to question the [3]-coordination of $\mathrm{Li}^{+}$in this structure.

${ }^{[7]} \mathrm{Li}^{+}$was found in only two coordination polyhedra (from two different structures) and ${ }^{[8]} \mathrm{Li}^{+}$was found in only one coordination polyhedron. ${ }^{[8]} \mathrm{Li}^{+}$was reported in $\mathrm{Rb}_{6} \mathrm{LiPr}_{11} \mathrm{Cl}_{16}\left[\mathrm{SeO}_{3}\right]_{12}$ (Lipp \& Schleid, 2006) with eight symmetrically equivalent bonds of length $2.513 \AA$ giving an incident bond-valence sum of $0.835 \mathrm{v}$.u. with the parameters of Gagné \& Hawthorne (2015). The $U_{\text {eq }}$ value is five times that of its nearest-neighbour anions, all of which are equivalent, and twice that of the $\mathrm{Rb}^{+}$cations in the structure. ${ }^{[7]} \mathrm{Li}^{+}$has been reported in $\mathrm{LiGd}_{6} \mathrm{O}_{5}\left(\mathrm{BO}_{3}\right)_{3}$ (Chaminade et al., 1999) and $\mathrm{Li}_{2}(\mathrm{Mg}, \mathrm{Cu}) \mathrm{Cu}_{2}\left[\mathrm{Si}_{2} \mathrm{O}_{6}\right]_{2}$ (Horiuchi et al., 1997), with mean bond lengths of 2.337 and $2.325 \AA$ and incident bond-valence sums of 1.021 and 1.067 v.u., respectively. Moreover, there are no significant hiati in the list of increasing bond distances for each structure. $\mathrm{In} \mathrm{LiGd}_{6} \mathrm{O}_{5}\left(\mathrm{BO}_{3}\right)_{3}, U_{\text {eq }}$ for $\mathrm{Li}^{+}$is three-four times the values of the other atoms. In $\mathrm{Li}_{2}(\mathrm{Mg}, \mathrm{Cu}) \mathrm{Cu}_{2}\left[\mathrm{Si}_{2} \mathrm{O}_{6}\right]_{2}$, $U_{\text {eq }}$ for $\mathrm{Li}^{+}$is five times the values of the other metal atoms and three times the values of the anions; moreover, the displacements for $\mathrm{Li}^{+}$are very anisotropic. There is no alternative to $\mathrm{Li}^{+}$in [7]- and [8]-coordination in these structures, but it is apparent from the very large $U_{\text {eq }}$ values that $\mathrm{Li}^{+}$is 'rattling around' in overly large holes in these structures (the 
Table 1

Bond-length statistics for the five common alkali-metal ions.

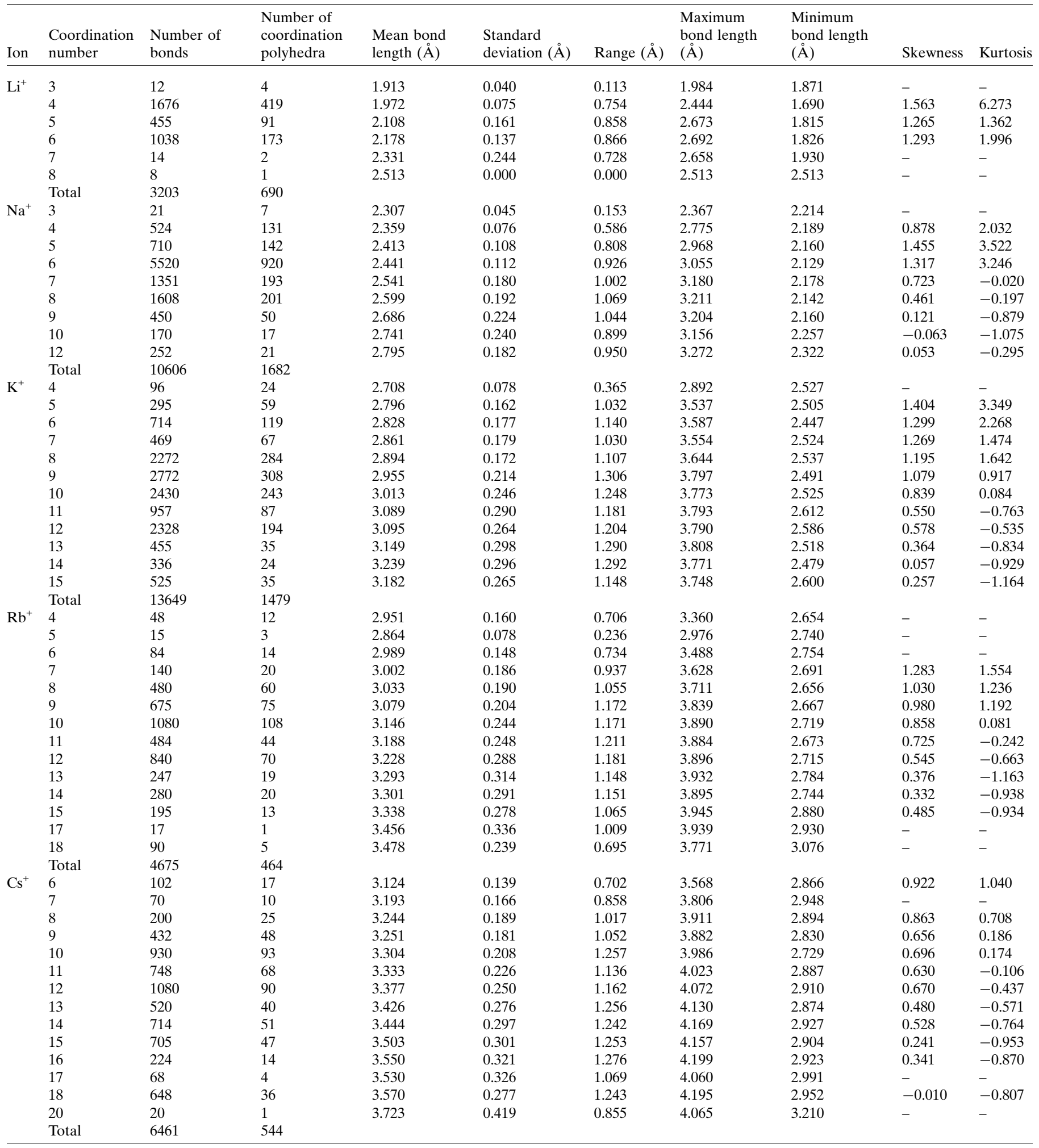

cation is significantly displaced from the centre of the polyhedron). Although one might argue that the resultant observed mean bond lengths are thus anomalous, $U_{\text {eq }}$ values are generally correlated with coordination number and it is difficult to refute the suggestion that what we observe for [7]and [8]-coordinated $\mathrm{Li}^{+}$is just a (non-linear) extrapolation of behaviour at lower coordination numbers. This issue is discussed further in $\S 10.3$. 
7.1.2. $\mathrm{Na}^{+} \cdot \mathrm{Na}^{+}$is observed in nine coordinations, from [3] to [12] excluding [11]. ${ }^{[6]} \mathrm{Na}^{+}$is by far the most common coordination $(n=920)$, followed by ${ }^{[8]} \mathrm{Na}^{+}(n=201)$ and ${ }^{[7]} \mathrm{Na}^{+}$ $(n=193) .{ }^{[3]} \mathrm{Na}^{+}(n=7)$ has a very small spread in bond lengths, from 2.214 to $2.367 \AA$, but this is a common feature we observe for all [3]-coordinated cations. The incident bondvalence sums are very low ( 0.70 v.u. on average); for example, in $\mathrm{Na}_{6} \mathrm{CoO}_{3}$ (Möller, 1998), the $\left\langle{ }^{[3]} \mathrm{Na}^{+}-\mathrm{O}^{2-}\right\rangle$ distance is $2.255 \AA$ (the shortest of the eight coordination polyhedra), and the incident bond-valence sum is 0.804 v.u. In this struc-

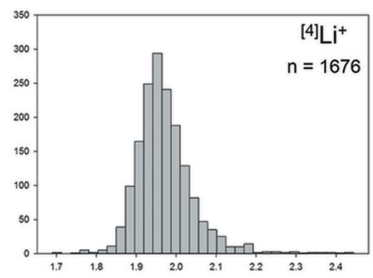

(a)

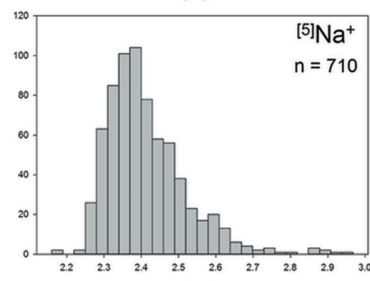

(e)

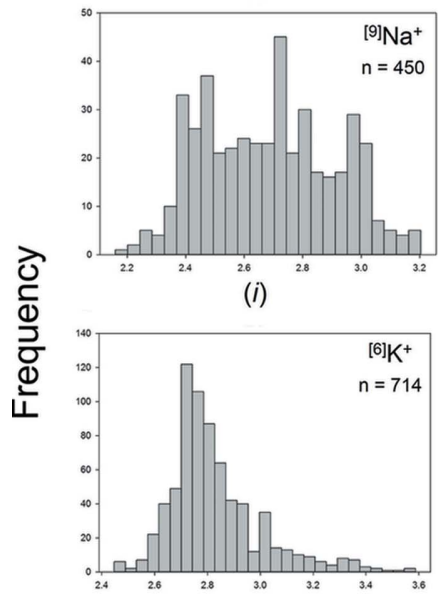

(m)

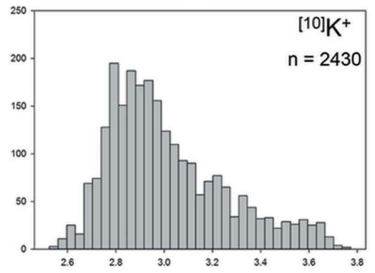

(q)

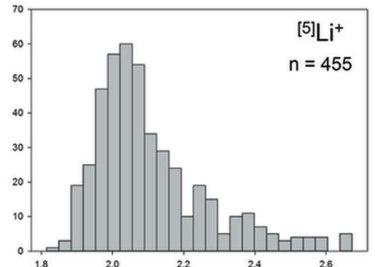

(b)

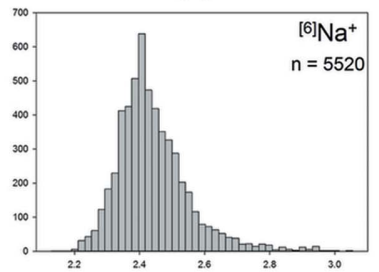

$(f)$

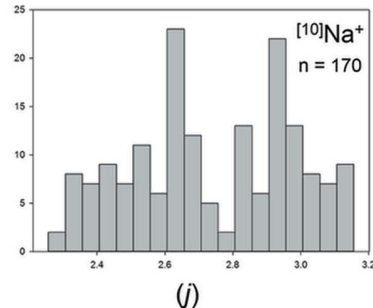

(j)

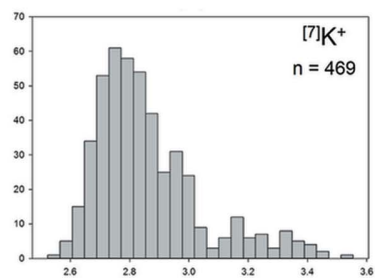

(n)

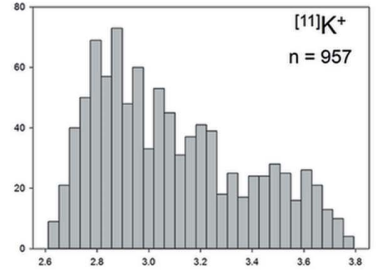

(r)

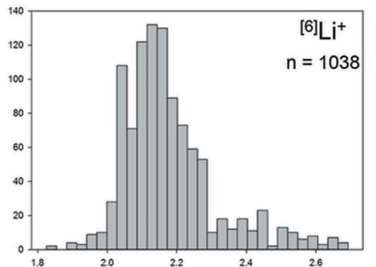

(c)

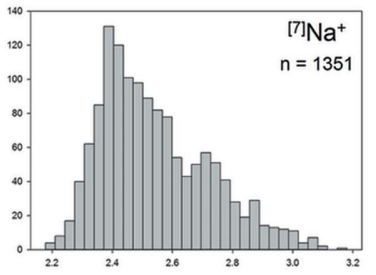

(g)

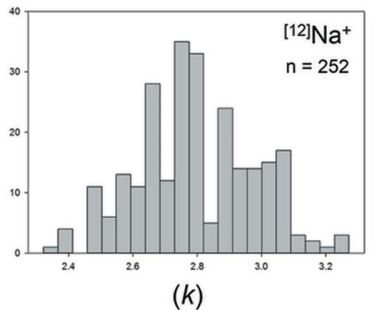

(k)

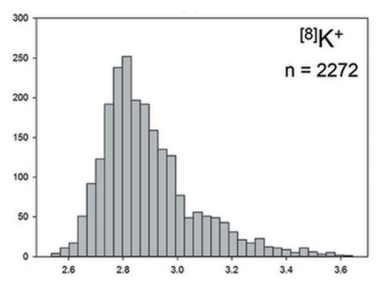

(o)

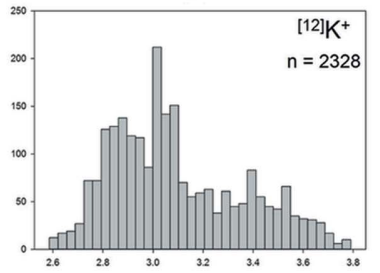

$(s)$

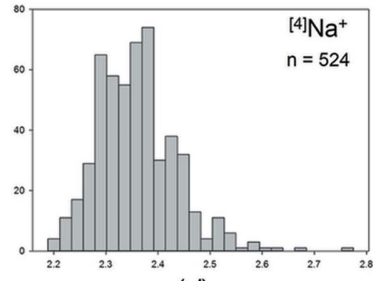

(d)

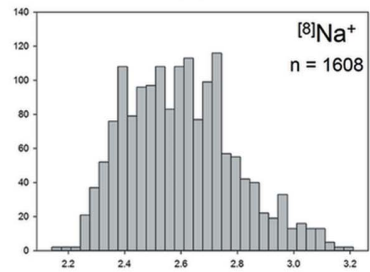

(h)

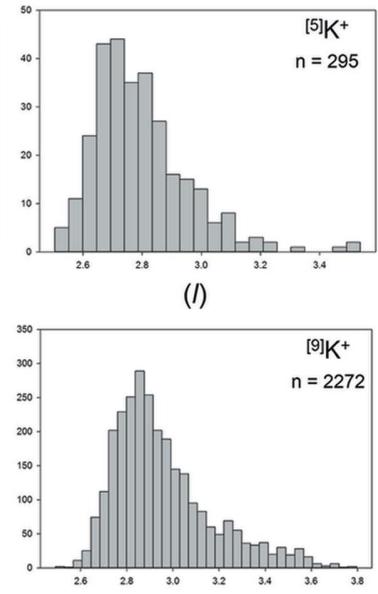

(p)

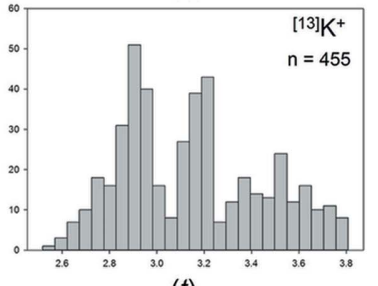

(t)

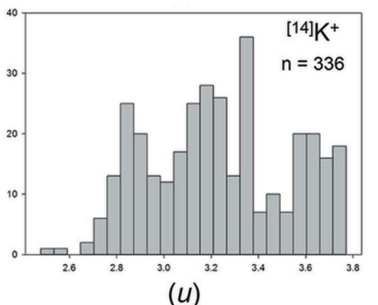

Bond length $(\AA)$

Figure 5

Bond-length distributions for the configurations of the alkali-metal ions bonded to $\mathrm{O}^{2-}$ with a sample size of $100+$ bonds: $(a)^{[4]} \mathrm{Li}^{+},(b){ }^{[5]} \mathrm{Li}^{+},(c){ }^{[6]} \mathrm{Li}^{+}$, $(d){ }^{[4]} \mathrm{Na}^{+},(e){ }^{[5]} \mathrm{Na}^{+},(f){ }^{[6]} \mathrm{Na}^{+},(g){ }^{[7]} \mathrm{Na}^{+},(h){ }^{[8]} \mathrm{Na}^{+},(i){ }^{[9]} \mathrm{Na}^{+},(j){ }^{[10]} \mathrm{Na}^{+},(k){ }^{[12]} \mathrm{Na}^{+},(l){ }^{[5]} \mathrm{K}^{+},(m){ }^{[6]} \mathrm{K}^{+},(n){ }^{[7]} \mathrm{K}^{+},(o){ }^{[8]} \mathrm{K}^{+},(p){ }^{[9]} \mathrm{K}^{+},(q){ }^{[10]} \mathrm{K}^{+},(r){ }^{[11]} \mathrm{K}^{+}$, $(s){ }^{[12]} \mathrm{K}^{+},(t){ }^{[13]} \mathrm{K}^{+},(u){ }^{[14]} \mathrm{K}^{+}$. 
ture, the coordination of ${ }^{[3]} \mathrm{Na}^{+}$is triangular with a $\left\langle\mathrm{O}^{2-}-\right.$ $\left.\mathrm{Na}^{+}-\mathrm{O}^{2-}\right\rangle$ angle of $117.2^{\circ}$ and a slight pyramidal character, and the next-nearest $\mathrm{O}^{2-}$ anion is at $3.57 \AA$, far beyond any significant bond-valence interaction. Thus the occurrence of
$\mathrm{Na}^{+}$in [3]-coordination seems established. For [12] coordination, the central cation and the coordinating anions generally show similar values of $U_{\text {eq }}$ with no anomalous disorder.

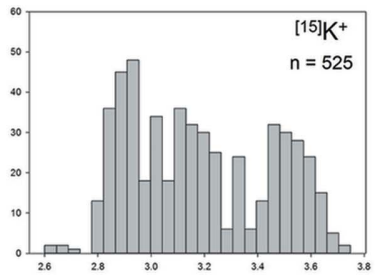

(v)

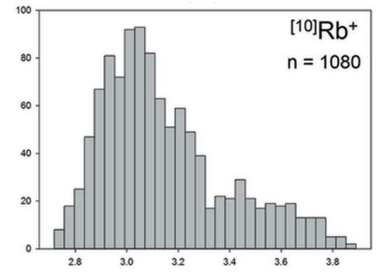

$(z)$

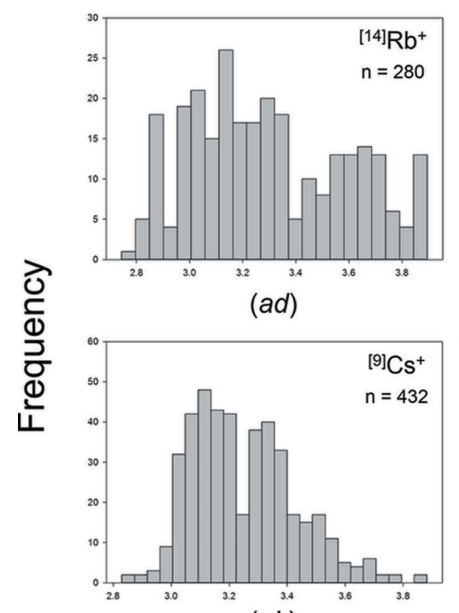

$(a h)$

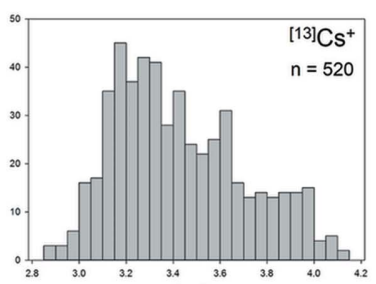

(al)

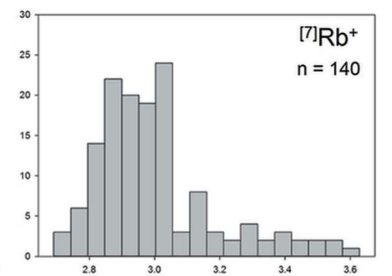

$(w)$

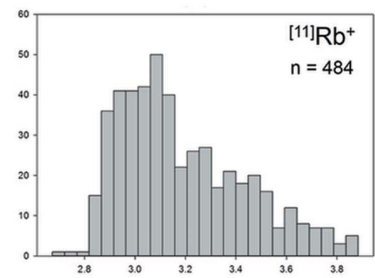

(aa)

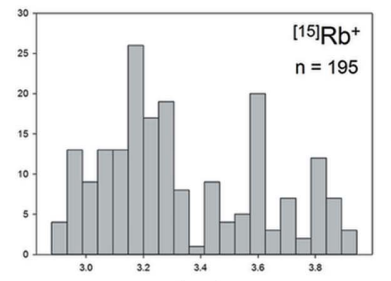

(ae)

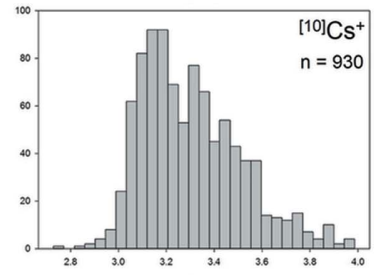

(ai)

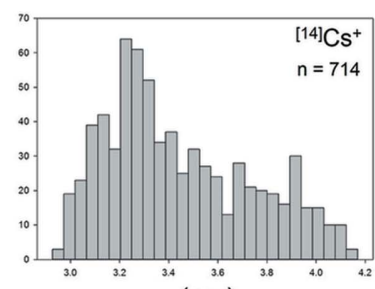

(am)

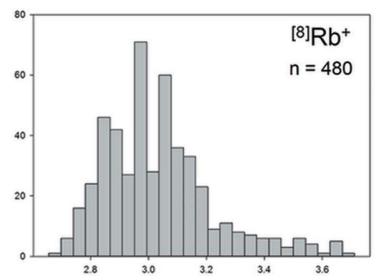

$(x)$

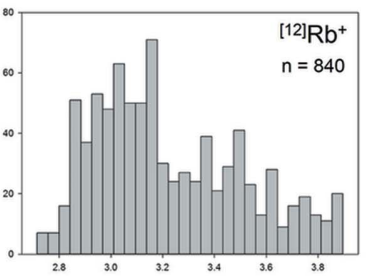

$(a b)$

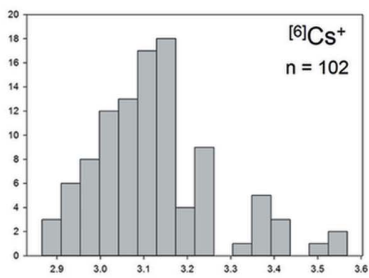

(af)

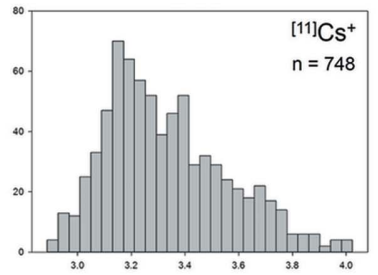

(aj)

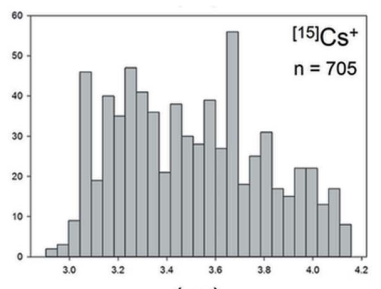

(an)

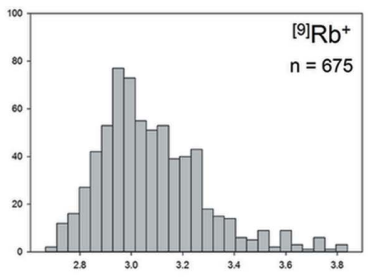

(y)

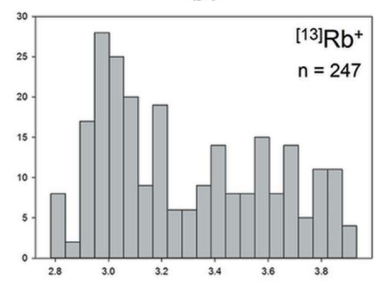

(ac)

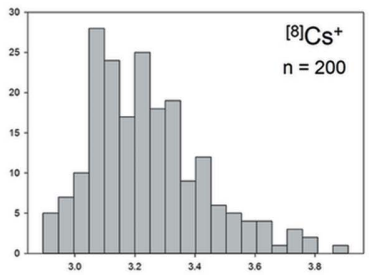

(ag)

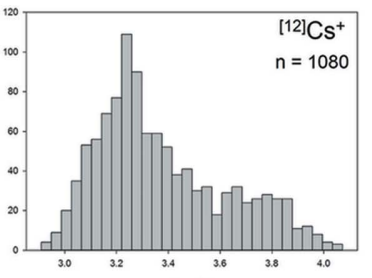

(ak)

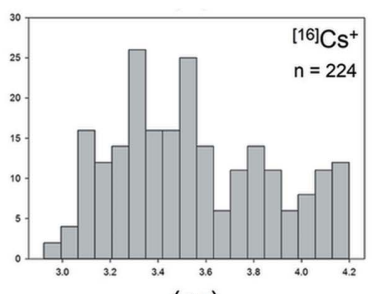

(ao)

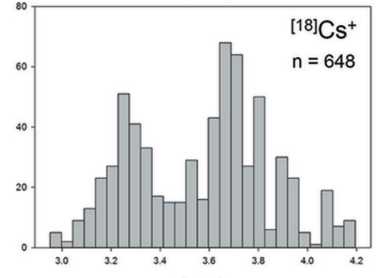

(ap)

Figure 5 (continued)

Bond length $(\AA)$

Bond-length distributions for the configurations of the alkali-metal ions bonded to $\mathrm{O}^{2-}$ with a sample size of $100+$ bonds: $(v)^{[15]} \mathrm{K}^{+},(w){ }^{[7]} \mathrm{Rb}^{+},(x)^{[8]} \mathrm{Rb}^{+}$, (y) ${ }^{[9]} \mathrm{Rb}^{+},(z){ }^{[10]} \mathrm{Rb}^{+},(a a){ }^{[11]} \mathrm{Rb}^{+},(a b){ }^{[12]} \mathrm{Rb}^{+},(a c){ }^{[13]} \mathrm{Rb}^{+},(a d){ }^{[14]} \mathrm{Rb}^{+},(a e){ }^{[15]} \mathrm{Rb}^{+},(a f){ }^{[6]} \mathrm{Cs}^{+},(a g){ }^{[8]} \mathrm{Cs}^{+},(a h){ }^{[9]} \mathrm{Cs}^{+},(a i){ }^{[10]} \mathrm{Cs}^{+},(a j){ }^{[11]} \mathrm{Cs}^{+},(a k)$ ${ }^{[12]} \mathrm{Cs}^{+},($al $){ }^{[13]} \mathrm{Cs}^{+},($am $){ }^{[14]} \mathrm{Cs}^{+},($an $){ }^{[15]} \mathrm{Cs}^{+},($ao $){ }^{[16]} \mathrm{Cs}^{+},($ap $){ }^{[18]} \mathrm{Cs}^{+}$. 


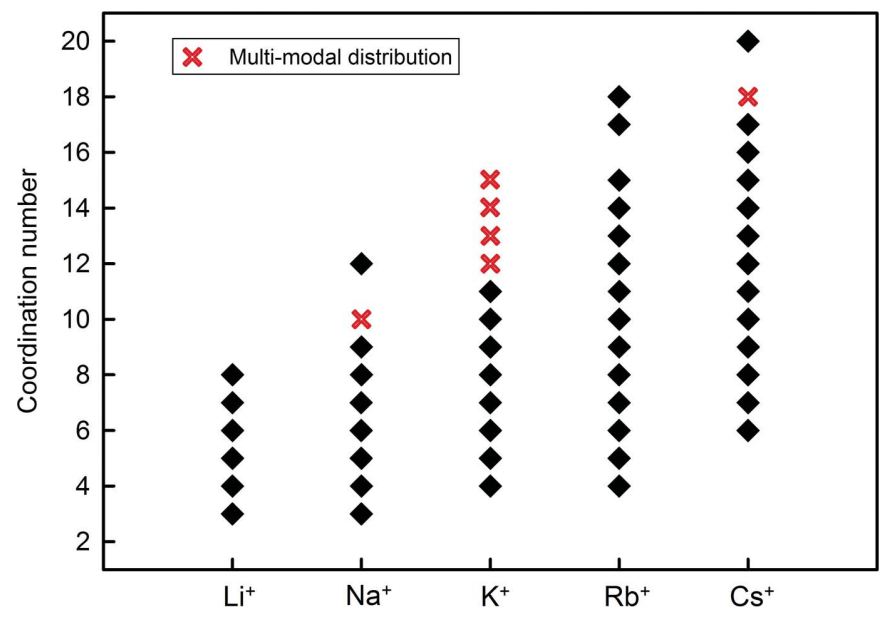

Figure 6

Observed coordination numbers for the alkali-metal ions. Multi-modal distributions are identified.

7.1.3. $\mathrm{K}^{+} . \mathrm{K}^{+}$is observed in 12 different coordinations, from [4] to [15], with a preference for coordination numbers $9(n=$ $308), 8(n=284)$ and $10(n=243)$. There are 24 coordination polyhedra for ${ }^{[4]} \mathrm{K}$ and the central cation and the coordinating anions generally show similar values of $U_{\text {eq }}$ with no anomalous disorder. The grand mean incident bond-valence for ${ }^{[4]} \mathrm{K}$ is 0.77 v.u. with a range of $0.53-0.92$ v.u.

7.1.4. $\mathbf{R b}^{+} . \mathrm{Rb}^{+}$is observed in 14 coordinations from [4] to [18], excluding [16]. It favourably adopts coordination number $10(n=108)$, followed by coordination numbers $9(n=75)$ and $8(n=60)$. Despite little data, ${ }^{[4]} \mathrm{Rb}^{+}(n=12)$ shows a typical

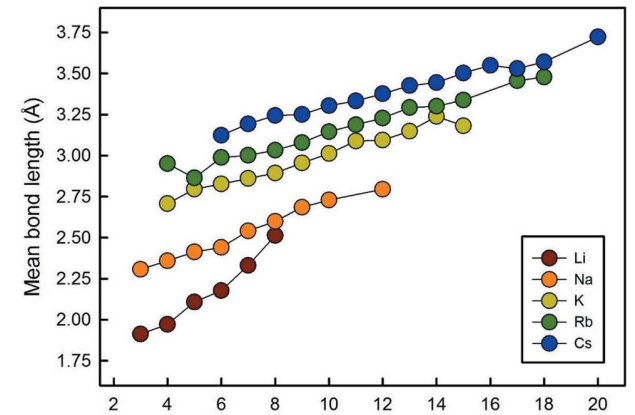

(a)

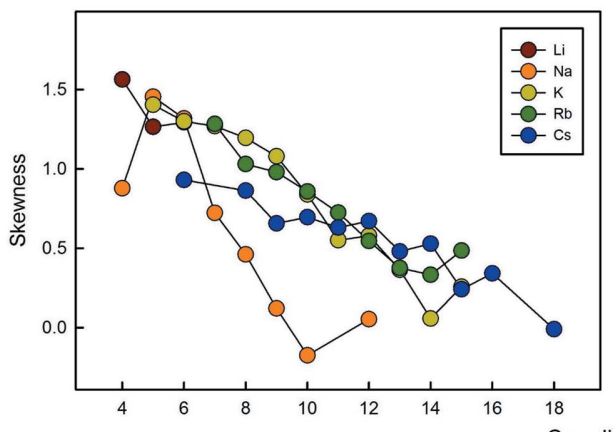

(c)

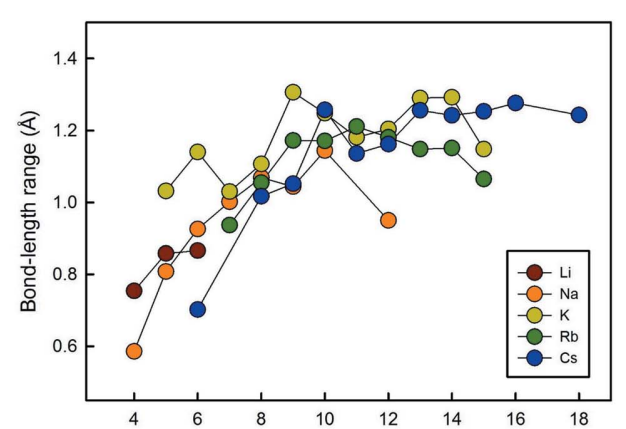

(b)

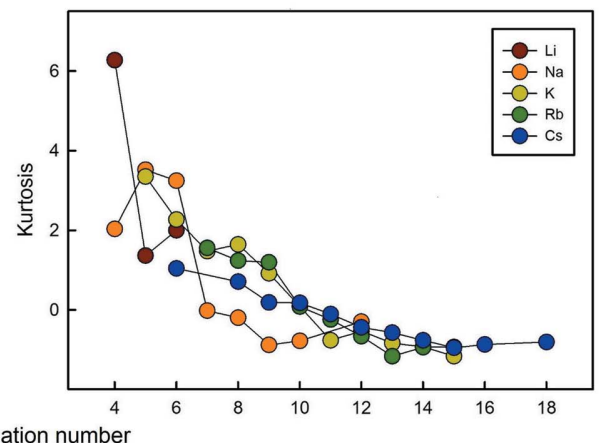

(d)
Figure 7

Values of $(a)$ grand mean bond length, $(b)$ bond-length range, $(c)$ skewness and $(d)$ kurtosis for the different coordination numbers of the alkali-metal ions distribution and the constituent atoms are well behaved in most of the structure refinements. The grand mean incident bond valence for ${ }^{[4]} \mathrm{Rb}^{+}$is 0.57 v.u. with a range of $0.49-$ 0.65 v.u. The following coordination numbers [15] $(n=13)$, [17] $(n=1)$ and [18] $(n=5)$ have mean incident bond-valence sums of $0.947,1.002$ and 0.912 v.u., respectively. The $U_{\text {eq }}$ values tend to be very high for the central cations in these structures. For example, in $\mathrm{Rb}_{5} \mathrm{VONb}_{14} \mathrm{O}_{38}$ (Haddad \& Jouini, 1997), $U_{\text {eq }}$ of ${ }^{[18]} \mathrm{Rb}^{+}$is $5-7$ times those of the coordinating anions; in $\mathrm{Rb}_{10} \mathrm{Ta}_{29.2} \mathrm{O}_{78}$ (Fallon \& Gatehouse, 1980), $U_{\text {eq }}$ of ${ }^{[18]} \mathrm{Rb}^{+}$is 15-20 times those of the coordinating anions; in $\mathrm{Rb}_{2} \mathrm{~V}_{3} \mathrm{P}_{4} \mathrm{O}_{17}$ (Lii et al., 1990), $U_{\text {eq }}$ of ${ }^{[17]} \mathrm{Rb}^{+}$is $\sim 3$ times those of the coordinating anions.

7.1.5. $\mathrm{Cs}^{+} \cdot \mathrm{Cs}^{+}$is observed in 14 coordinations, from [6] to [20] excluding [19], with a preference for coordination numbers $10(n=94)$ and $12(n=90) .{ }^{[6]} \mathrm{Cs}^{+}(n=18)$ and ${ }^{[7]} \mathrm{Cs}^{+}$ $(n=10)$ show regular distributions despite limited data, and the central cations and the coordinating anions generally show similar values of $U_{\text {eq }}$ with no anomalous disorder. For ${ }^{[20]} \mathrm{Cs}^{+}$, there are distances $3.210 \times 8$ and $4.064 \times 12 \AA$ with incident bond-valence sums of 0.886 v.u. for [8] and 1.029 v.u. for [20], and the $U_{\text {eq }}$ value of the central $\mathrm{Cs}^{+}$is $\sim 5$ times those of the coordinating anions.

\subsection{Grand mean bond length as a function of coordination number}

Fig. 7(a) shows the variation in mean bond length as a function of coordination number, and is given for all configurations regardless of sample size. The correlation is positive and is surprisingly regular. Minor anomalies $\left(\right.$ e.g. $\left.{ }^{[5]} \mathrm{Rb}^{+}\right)$can be attributed to a small sample size. We note that the slope of the variation for each ion in Fig. 7(a) decreases slightly with the increasing size of the cations.

\subsection{Range in bond length as a function of coordination number}

Fig. 7(b) shows the variation in the range of bond lengths as a function of coordination number. As the ranges are strongly dependent on sample size, we omitted data for configurations of less than 100 bonds. Although this criterion is sufficient to remove major outliers, the value for ${ }^{[12]} \mathrm{Na}^{+}$is $\sim 0.22 \AA$, smaller than expected from the general trend of Fig. 7(b); the reason for this difference is not clear. There is a strong non-linear trend in Fig. 7(b); the range in bond lengths is positively correlated with coordination number, and the range increases more rapidly at lower coordination numbers, and 
Table 2

Bond-length statistics for the five common alkaline-earth-metal ions.

\begin{tabular}{|c|c|c|c|c|c|c|c|c|c|c|}
\hline Ion & $\begin{array}{l}\text { Coordination } \\
\text { number }\end{array}$ & $\begin{array}{l}\text { Number of } \\
\text { bonds }\end{array}$ & $\begin{array}{l}\text { Number of } \\
\text { coordination } \\
\text { polyhedra }\end{array}$ & $\begin{array}{l}\text { Mean bond } \\
\text { length }(\AA)\end{array}$ & $\begin{array}{l}\text { Standard } \\
\text { deviation }(\AA)\end{array}$ & Range (̊̊) & $\begin{array}{l}\text { Maximum } \\
\text { bond length } \\
(\AA)\end{array}$ & $\begin{array}{l}\text { Minimum } \\
\text { bond length } \\
(\AA)\end{array}$ & Skewness & Kurtosis \\
\hline \multirow[t]{3}{*}{$\mathrm{Be}^{2+}$} & 3 & 24 & 8 & 1.550 & 0.018 & 0.081 & 1.587 & 1.506 & - & - \\
\hline & 4 & 644 & 161 & 1.637 & 0.040 & 0.346 & 1.887 & 1.541 & 1.988 & 8.529 \\
\hline & Total & 668 & 169 & & & & & & & \\
\hline \multirow[t]{5}{*}{$\mathrm{Mg}^{2+}$} & 4 & 48 & 12 & 1.939 & 0.020 & 0.068 & 1.977 & 1.909 & - & - \\
\hline & 5 & 120 & 24 & 2.044 & 0.066 & 0.342 & 2.249 & 1.907 & 0.643 & 0.271 \\
\hline & 6 & 2556 & 426 & 2.089 & 0.059 & 0.562 & 2.497 & 1.935 & 1.976 & 8.608 \\
\hline & 8 & 56 & 7 & 2.255 & 0.122 & 0.568 & 2.582 & 2.014 & - & - \\
\hline & Total & 2780 & 469 & & & & & & & \\
\hline \multirow[t]{8}{*}{$\mathrm{Ca}^{2+}$} & 6 & 1266 & 211 & 2.371 & 0.069 & 0.637 & 2.847 & 2.210 & 1.636 & 5.908 \\
\hline & 7 & 2009 & 287 & 2.448 & 0.133 & 1.016 & 3.140 & 2.124 & 1.828 & 4.607 \\
\hline & 8 & 4152 & 519 & 2.498 & 0.151 & 1.027 & 3.176 & 2.149 & 1.430 & 2.739 \\
\hline & 9 & 1035 & 115 & 2.559 & 0.196 & 0.956 & 3.197 & 2.241 & 1.247 & 0.939 \\
\hline & 10 & 160 & 16 & 2.632 & 0.215 & 0.902 & 3.122 & 2.220 & 0.478 & -0.400 \\
\hline & 11 & 77 & 7 & 2.614 & 0.177 & 0.686 & 2.965 & 2.279 & - & - \\
\hline & 12 & 156 & 13 & 2.668 & 0.175 & 0.791 & 3.117 & 2.326 & 0.724 & 0.379 \\
\hline & Total & 8855 & 1168 & & & & & & & \\
\hline \multirow[t]{8}{*}{$\mathrm{Sr}^{2+}$} & 6 & 78 & 13 & 2.477 & 0.050 & 0.244 & 2.591 & 2.347 & - & - \\
\hline & 7 & 266 & 38 & 2.638 & 0.151 & 0.937 & 3.306 & 2.369 & 1.553 & 3.693 \\
\hline & 8 & 904 & 113 & 2.656 & 0.163 & 1.019 & 3.368 & 2.349 & 1.711 & 3.740 \\
\hline & 9 & 909 & 101 & 2.704 & 0.178 & 1.088 & 3.397 & 2.309 & 1.545 & 3.094 \\
\hline & 10 & 500 & 50 & 2.769 & 0.213 & 1.033 & 3.399 & 2.366 & 0.962 & 0.456 \\
\hline & 11 & 88 & 8 & 2.798 & 0.198 & 0.837 & 3.319 & 2.482 & - & - \\
\hline & 12 & 636 & 53 & 2.825 & 0.181 & 0.953 & 3.358 & 2.405 & 0.744 & 0.613 \\
\hline & Total & 3381 & 376 & & & & & & & \\
\hline \multirow[t]{10}{*}{$\mathrm{Ba}^{2+}$} & 6 & 126 & 21 & 2.689 & 0.094 & 0.400 & 2.866 & 2.466 & -0.404 & -0.665 \\
\hline & 7 & 147 & 21 & 2.792 & 0.135 & 0.855 & 3.369 & 2.514 & 1.133 & 2.916 \\
\hline & 8 & 704 & 88 & 2.816 & 0.129 & 0.846 & 3.376 & 2.530 & 0.962 & 1.273 \\
\hline & 9 & 1278 & 142 & 2.860 & 0.154 & 1.074 & 3.554 & 2.480 & 1.000 & 1.330 \\
\hline & 10 & 1930 & 193 & 2.915 & 0.155 & 1.014 & 3.498 & 2.484 & 0.783 & 1.072 \\
\hline & 11 & 902 & 82 & 2.944 & 0.181 & 1.023 & 3.612 & 2.589 & 0.667 & 0.074 \\
\hline & 12 & 3624 & 302 & 2.965 & 0.152 & 0.988 & 3.624 & 2.636 & 1.239 & 1.951 \\
\hline & 13 & 78 & 6 & 3.010 & 0.206 & 0.922 & 3.463 & 2.541 & - & - \\
\hline & 14 & 14 & 1 & 3.080 & 0.242 & 0.879 & 3.553 & 2.674 & - & - \\
\hline & Total & 8813 & 856 & & & & & & & \\
\hline
\end{tabular}

flattens out at higher coordination numbers.

\subsection{Skewness and kurtosis as a function of coordination number}

Figs. 7(c) and $(d)$ show skewness and kurtosis as a function of coordination number, respectively, for the five alkali-metal ions. As skewness and kurtosis are highly influenced by the amount of data used in their calculation, the graphs only show values for configurations with 100 bonds or more, as discussed above. Fig. 7(c) shows a more-or-less linear decrease in skewness with increasing coordination number. $\mathrm{Na}^{+}$shows a lower skewness for coordination [4] than would be expected from the trend, for reasons that are not clear, and the rate of decrease in kurtosis with increasing coordination number is greater than that for the other alkali-metal ions which show a surprisingly consistent trend (considering the sensitivity of skewness to sample size and outliers). Fig. $7(d)$ shows a well developed trend of non-linear decrease in kurtosis as a function of coordination number for all the alkali-metal ions. The trend for $\mathrm{Na}^{+}$is again somewhat less consistent than for the other ions, and the shape is similar to that exhibited for skewness (Fig. 7c).

\section{Results for the alkaline-earth metals}

Our collection and filtering criteria resulted in a combined sample size of 24487 bonds and 3038 coordination polyhedra. Table 2 gives the 29 observed configurations, the mean bond length and standard deviation, the minimum and maximum bond length (and range), the skewness and kurtosis (where justified by sample size), and the number of bonds and coordination polyhedra for the five common alkaline-earth-metal ions. Fig. S2 gives all the bond-length distributions for the alkaline-earth metals; those with adequate sample sizes (as discussed above) are shown in Fig. 8. These ions are found in slightly more than half the number of configurations observed for the alkali metals (55), primarily because these ions are not observed in coordinations higher than [12], with the exception of $\mathrm{Ba}^{2+}$ (observed as [13] and [14]).

\subsection{Observed coordination numbers}

Fig. 9 shows the variation of the coordination numbers for the alkaline-earth-metal ions. The minimum and maximum coordination numbers increase with size, [3] and [4] for $\mathrm{Be}^{2+}$, [4] and [8] for $\mathrm{Mg}^{2+},[6]$ and [12] for $\mathrm{Ca}^{2+}$ and $\mathrm{Sr}^{2+}$, and [6] and [14] for $\mathrm{Ba}^{2+}$. The number of coordination numbers also increases along the series, from 2 for $\mathrm{Be}^{2+}$ to 4 for $\mathrm{Mg}^{2+}, 7$ for $\mathrm{Ca}^{2+}, 8$ for $\mathrm{Sr}^{2+}$ and 9 for $\mathrm{Ba}^{2+}$. 
8.1.1. $\mathrm{Be}^{2+} . \mathrm{Be}^{2+}$ occurs in two coordination numbers, with a clear preference for coordination number [4] $(n=161)$ compared with its 3-coordinated form ${ }^{[3]} \mathrm{Be}^{2+}(n=8)$. However, the coordination of [3] seems well established for Be. For example, Leoni et al. (2005) report the structure of $\mathrm{Ba}_{3}\left[\mathrm{Be}_{5} \mathrm{O}_{8}\right]$ with a $\mathrm{Be}$ atom [3]-coordinated at distances of
1.545 (9) and 1.561 (5) $\times 2 \AA$ with well behaved $U_{\text {eq }}$ values for both the central cation and the coordinating anions, and an incident bond-valence sum of 1.96 v.u.

8.1.2. $\mathrm{Mg}^{2+} \cdot \mathrm{Mg}^{2+}$ occurs in four different coordination numbers from [4] to [8], excluding [7]. ${ }^{[6]} \mathrm{Mg}^{2+}$ is by far the most common coordination with $n=426$ coordination poly-

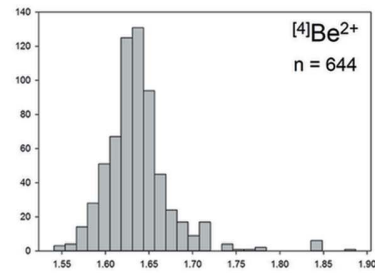

(a)

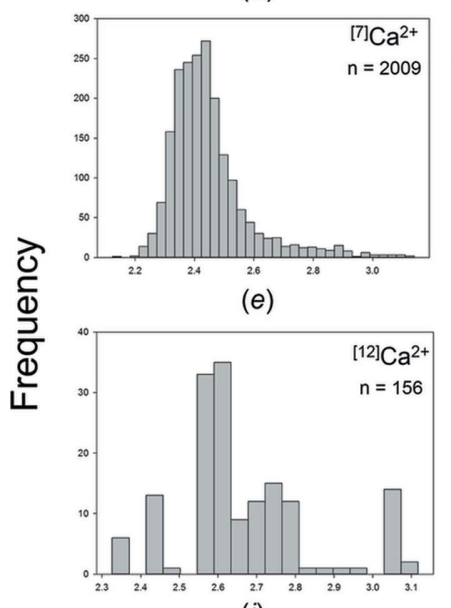

(i)

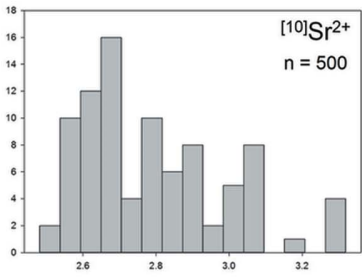

(m)

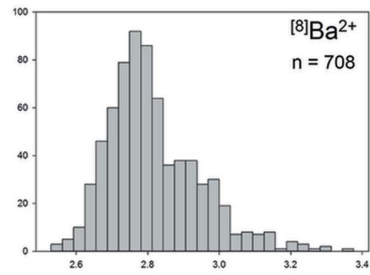

(q)

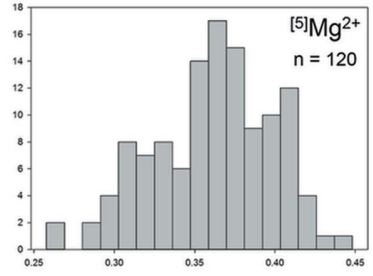

(b)
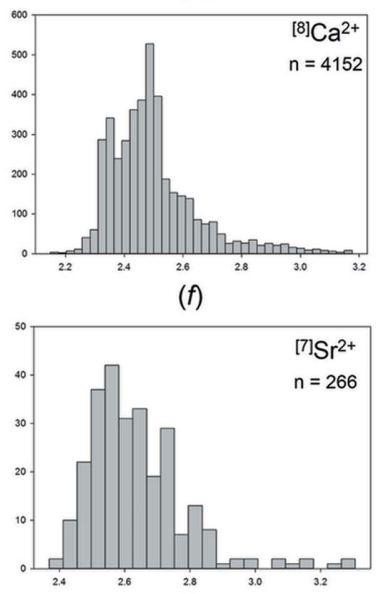

(j)

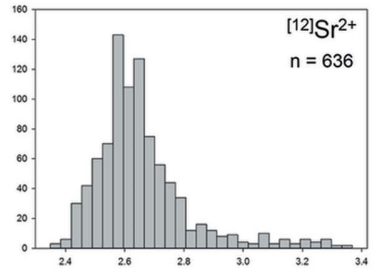

(n)

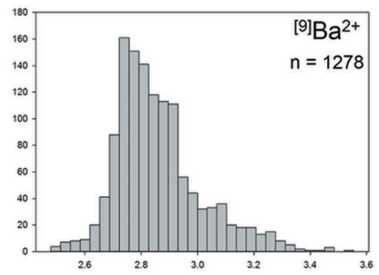

(r)

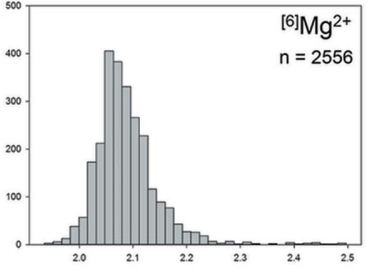

(c)

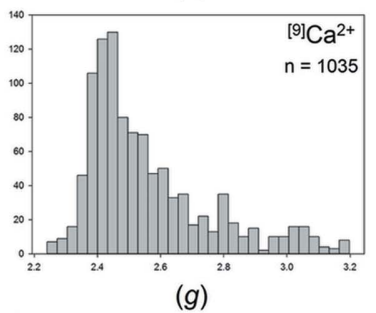

(g)

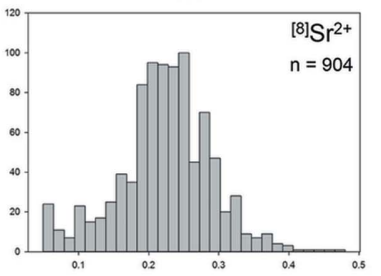

(k)

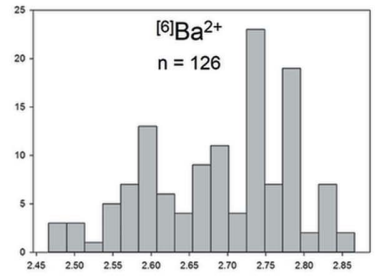

(O)

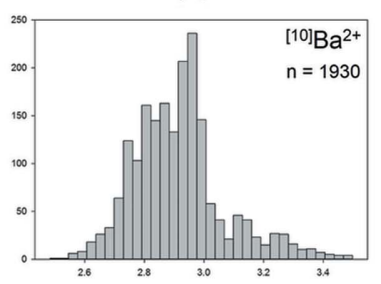

(s)

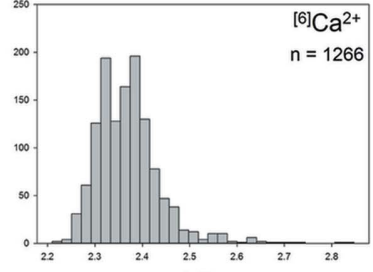

(d)

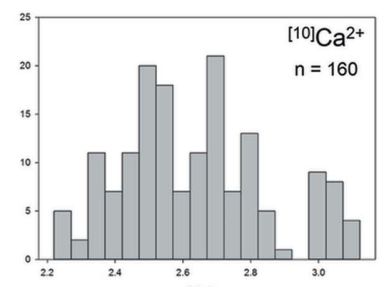

(h)

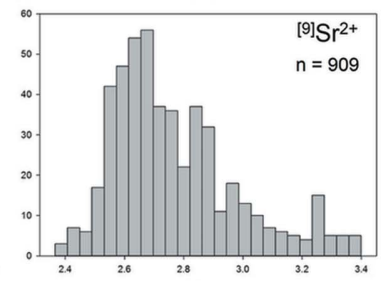

(I)

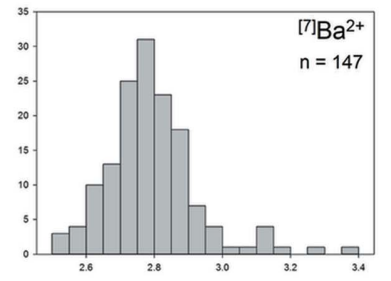

(p)

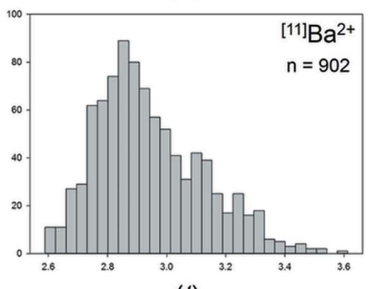

(t)

Figure 8

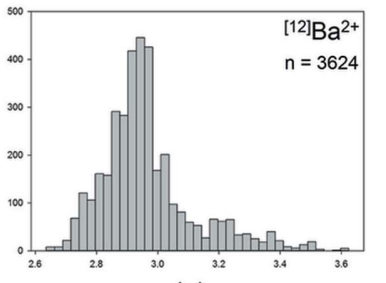

(u)

Bond-length distributions for the configurations of the alkaline-earth-metal ions bonded to $\mathrm{O}^{2-}$ with a sample size of $100+$ bonds: $(a)^{[4]} \mathrm{Be}^{2+},(b)^{[5]} \mathrm{Mg}^{2+}$, $\left.(c){ }^{[6]} \mathrm{Mg}^{2+},(d){ }^{[6]} \mathrm{Ca}^{2+},(e){ }^{[7]} \mathrm{Ca}^{2+},(f){ }^{[8]} \mathrm{Ca}^{2+},(g)\right)^{[9]} \mathrm{Ca}^{2+},(h)^{[10]} \mathrm{Ca}^{2+},(i){ }^{[12]} \mathrm{Ca}^{2+},(j){ }^{[7]} \mathrm{Sr}^{2+},(k)^{[8]} \mathrm{Sr}^{2+},(l){ }^{[9]} \mathrm{Sr}^{2+},(m){ }^{[10]} \mathrm{Sr}^{2+},(n)^{[12]} \mathrm{Sr}^{2+},(o){ }^{[6]} \mathrm{Ba}^{2+},(p)$ ${ }^{[7]} \mathrm{Ba}^{2+},(q){ }^{[8]} \mathrm{Ba}^{2+},(r){ }^{[9]} \mathrm{Ba}^{2+},(s){ }^{[10]} \mathrm{Ba}^{2+},(t){ }^{[11]} \mathrm{Ba}^{2+},(u){ }^{[12]} \mathrm{Ba}^{2+}$. 
hedra. ${ }^{[4]} \mathrm{Mg}^{2+}(n=12)$ has an apparent bimodal distribution, which is clearly an artifact of a small number of bond lengths that show little spread. However, the coordination [4] must be considered as well established for $\mathrm{Mg}^{2+}$ as structure refinements show well behaved cations and anions. The grand mean incident bond valence for ${ }^{[8]} \mathrm{Mg}$ is $1.93 \mathrm{v} . \mathrm{u}$. with a range of 1.78-2.20 v.u. The structures containing ${ }^{[8]} \mathrm{Mg}^{2+}$ look well refined, although $U_{\text {eq }}$ values are usually somewhat higher than those of the anions, suggesting significant displacement. This effect was also suggested by Shannon \& Rossman (1992) with

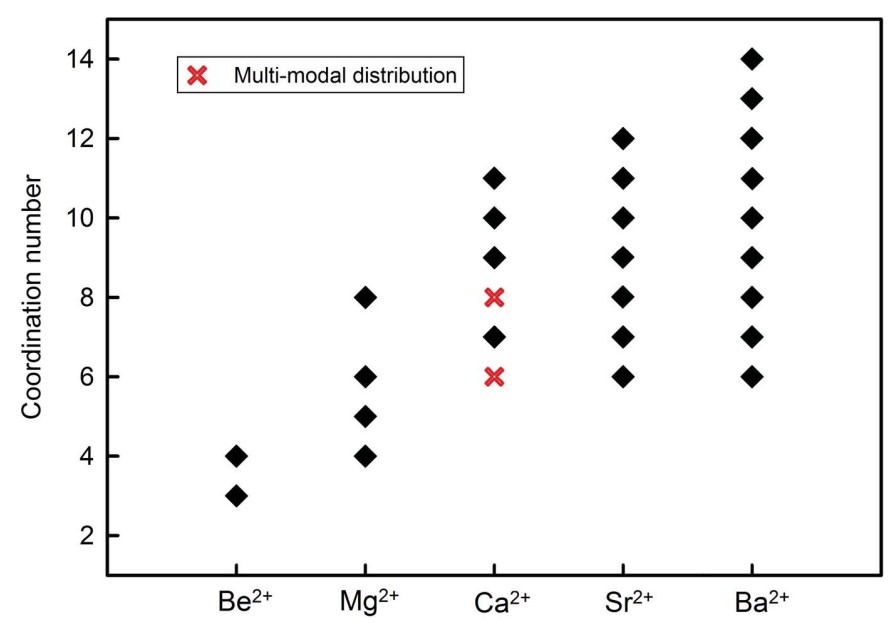

Figure 9

Observed coordination numbers for the alkaline-earth-metal ions. Multimodal distributions are identified.

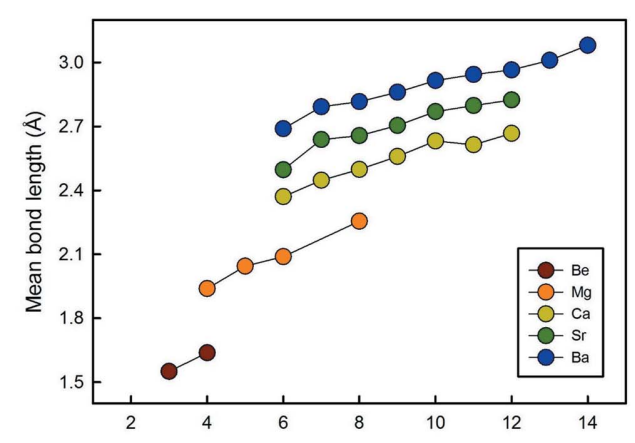

(a)

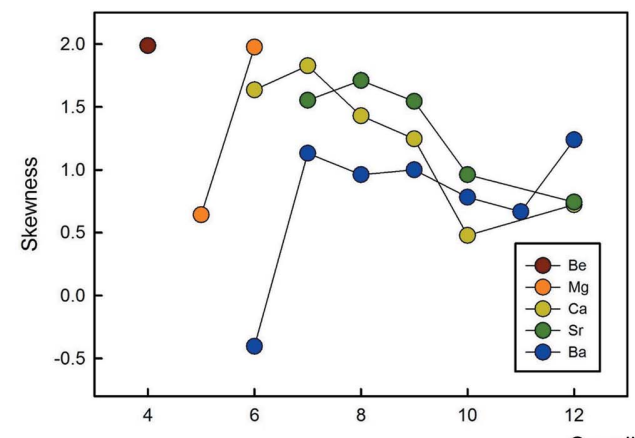

(c)

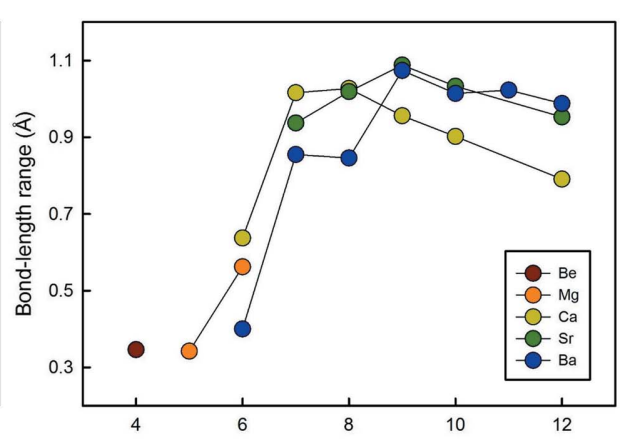

(b)

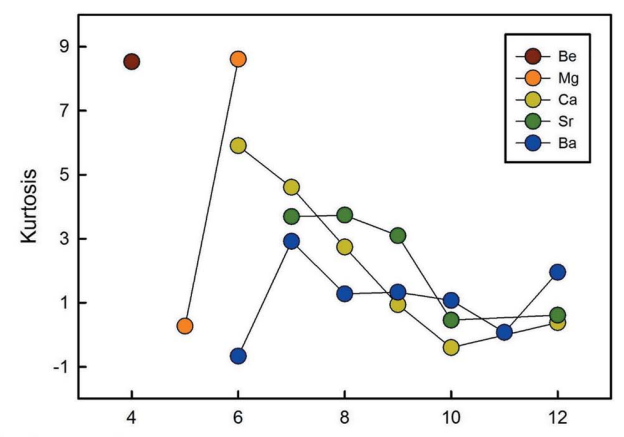

(d)
Figure 10

Values of $(a)$ grand mean bond length, $(b)$ bond-length range, $(c)$ skewness and $(d)$ kurtosis for the different coordination numbers of the alkaline-earth-metal ions. regard to deviations of measured and calculated dielectric constants for ${ }^{[8]} \mathrm{Mg}^{2+}$.

8.1.3. $\mathrm{Ca}^{2+} \cdot \mathrm{Ca}^{2+}$ occurs in seven different coordinations from [6] to [12] with a preference for coordination number [8] $(n=519)$. [6]-coordinated $\mathrm{Ca}^{2+}$ is well established $(n=211)$. The lowest observed $\left\langle{ }^{[6]} \mathrm{Ca}^{2+}-\mathrm{O}\right\rangle$ distance is 2.254 (4) $\AA$ in $\mathrm{Ba}_{3} \mathrm{CaRu}_{2} \mathrm{O}_{9}$ (Wilkens \& Müller-Buschbaum, 1991); this seems very short compared with the grand $\left\langle{ }^{[6]} \mathrm{Ca}^{2+}-\mathrm{O}\right\rangle$ distance of 2.371 (69) $\AA$ (Table 2), but there is no reason to reject this value based on the structure refinement. There are few examples of the higher coordination-numbers: ${ }^{[10]} \mathrm{Ca}(n=$ $16),{ }^{[11]} \mathrm{Ca}(n=7)$ and ${ }^{[12]} \mathrm{Ca}(n=13)$. The grand mean incident bond valence for ${ }^{[12]} \mathrm{Ca}$ is 2.03 v.u. with a range of $1.43-$ 2.50 v.u. The lowest values are for $\mathrm{Ca}_{3} \mathrm{Zn}_{4} \mathrm{Ti}_{16} \mathrm{O}_{38}$ (Gatehouse \& Grey, 1983) and $\mathrm{CaMg}_{2} \mathrm{Al}_{16} \mathrm{O}_{27}$ (Iyi et al., 1995). $\mathrm{Ca}_{3} \mathrm{Zn}_{4} \mathrm{Ti}_{16} \mathrm{O}_{38}$ has $\mathrm{Ca}-\mathrm{O}$ distances 2.762 (5) $\times 6$ and 2.792 (5) $\times 6 \AA$ with a mean value of $2.777 \AA$ and a displacement parameter that is 3-10 times those of the other atoms in the structure. $\mathrm{CaMg}_{2} \mathrm{Al}_{16} \mathrm{O}_{27}$ has $\mathrm{Ca}-\mathrm{O}$ distances $2.773(8) \times 6$ and $2.799(21) \times 6 \AA$ with a mean value of $2.786 \AA$ and a displacement parameter that is 2-6 times those of the other atoms in the structure. Thus the low incident bond-valence sums are associated with central cations that show very large displacements. The highest values are for $\mathrm{CaCu}_{3} \mathrm{Ge}_{4} \mathrm{O}_{12}$ (Ozaki et al., 1977) and $\mathrm{CaCu}_{3} \mathrm{Ge}_{4} \mathrm{O}_{12}$ (Chenevas et al., 1975). $\mathrm{CaCu}_{3} \mathrm{Ge}_{4} \mathrm{O}_{12}$ has $\mathrm{Ca}-\mathrm{O}$ distances of 2.549 (4) $\times 12 \AA$ and a displacement parameter that is similar to those of the other atoms in the structure. $\mathrm{CaCu}_{3} \mathrm{Ge}_{4} \mathrm{O}_{12}$ has $\mathrm{Ca}-\mathrm{O}$ distances of 2.562 (3) $\times 12 \AA$ and a displacement parameter that is similar to those of the other atoms in the structure. Thus the high incident bond-valence sums are associated with central cations that show displacements similar to those of the other atoms in the structure. 8.1.4. $\mathrm{Sr}^{2+} \cdot \mathrm{Sr}^{2+}$ occurs in 7 different coordinations from [6] to [12] with a preference for coordination numbers [8] $(n=113)$ and [9] $(n=101)$. An unusual coordination for $\mathrm{Sr}$ occurs in the crystal structure of $\quad \beta-\mathrm{Sr}_{10} \mathrm{Ga}_{6} \mathrm{O}_{19}$ (Kahlenburg, 2002), where one of 11 crystallographically distinct $\mathrm{Sr}$ atoms has bond lengths of 2.425 (8) $\times 2, \quad 2.471(9) \times 2$ and $3.350(9)$ $\times 2 \AA$; is $\mathrm{Sr}^{2+}[4]-$ or [6]-coordinated? For [4]-coordination, the four anions do not form a tetrahedron and the $\mathrm{Sr}^{2+}$ cation lies between the two closest anions with an $\mathrm{O}^{2-}-\mathrm{Sr}^{2+}-\mathrm{O}^{2-}$ angle of $175.4^{\circ}$. For [6]-coordination, the $\mathrm{Sr}^{2+}$ cation lies almost in the plane of four of the anions that form a face of the polyhedron. The sums of the incident bond valences are $\sim 1.5$ v.u. for both [4]- and [6]- 
coordination. The two next-nearest anions are $3.519 \AA$ away from the central $\mathrm{Sr}^{2+}$, but there are two $\mathrm{Ga}^{3+}$ atoms at $3.410 \AA$. Thus, the coordination in this particular case is uncertain, and the coordination polyhedron was omitted from the data for $\mathrm{Sr}^{2+}$.

8.1.5. $\mathrm{Ba}^{2+} . \mathrm{Ba}^{2+}$ occurs in nine different coordinations from [6] to [14] with a clear preference for coordination number [12] $(n=302)$. There are few examples of coordination numbers > [12]: ${ }^{[13]} \mathrm{Ba}^{2+}(n=6)$ and ${ }^{[14]} \mathrm{Ba}^{2+}(n=1)$. For ${ }^{[13]} \mathrm{Ba}^{2+}$, the sums of the incident bond valences are in the range $1.85-2.37$ v.u., and for ${ }^{[14]} \mathrm{Ba}^{2+}$ the sum of the incident bond valences is 1.98 v.u.

\subsection{Grand mean bond length as a function of coordination} number

Fig. 10(a) shows the variation in mean bond length as a function of coordination number, and is given for all configurations regardless of sample size. The correlation is positive and very regular. The slope of the variation for each ion of Fig. 10 (a) decreases slightly with the increasing size of the cations, although less than is the case for the alkali-metal ions.

\subsection{Range in bond length as a function of coordination number}

Fig. 10(b) shows the variation in the range of bond lengths as a function of coordination number, omitting data for configurations of less than 100 bonds. There is a strong nonlinear trend in the data; the range increases more rapidly at lower coordination numbers, [4]-[7], but levels out at $\sim$ [8] $(\sim 0.95 \pm 0.1 \AA)$ and is fairly constant thereafter, aside from a decrease for the higher coordination of $\mathrm{Ca}^{2+}$ (probably due to few data). The initial increase in range for smaller coordination numbers is steeper for the alkaline-earth metals than for the alkali metals (Fig. $7 b$ ) with mean slopes of 0.37 for $\mathrm{Mg}^{2+}$,

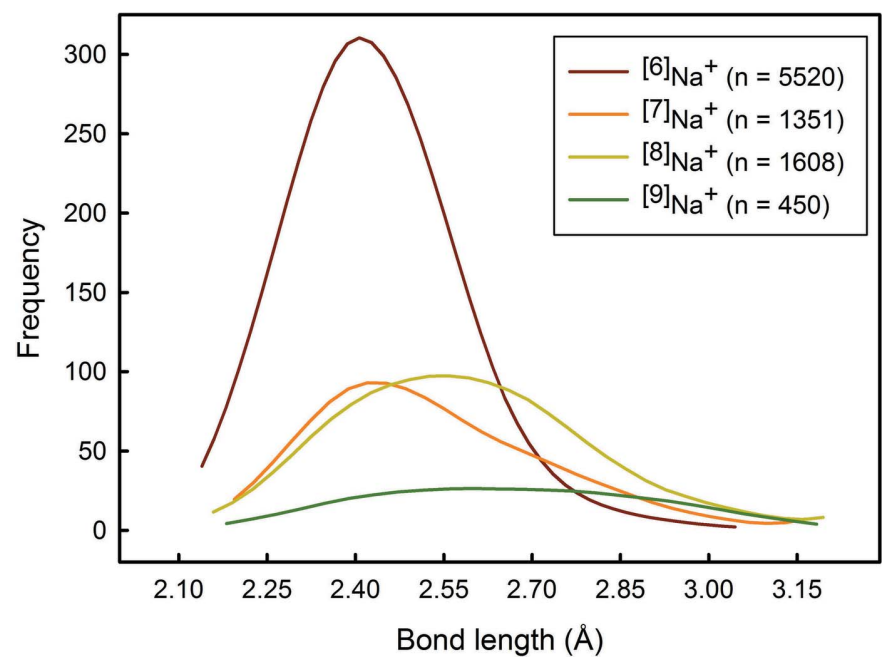

Figure 11

Kernel-density estimation of the bond-length distributions of the coordination numbers [6] to [9] for $\mathrm{Na}^{+}$. The plot shows that changes in skewness and kurtosis values are primarily due to a change in the shape of the maximum of the bond-length distribution.

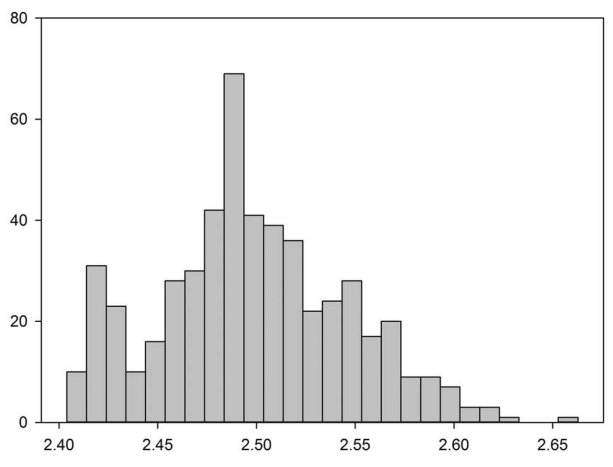

(a)

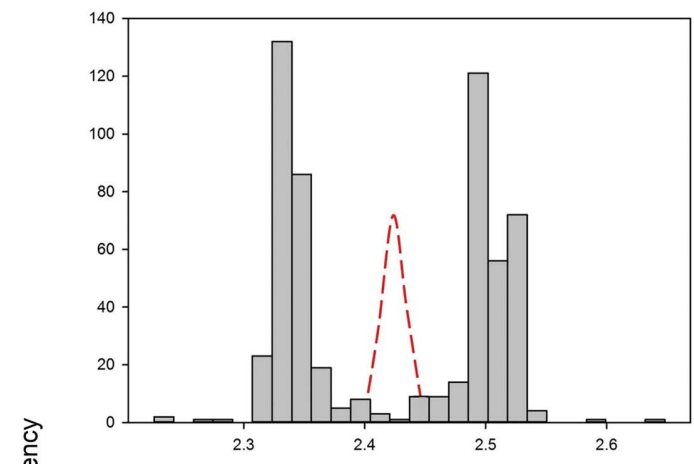

(b)

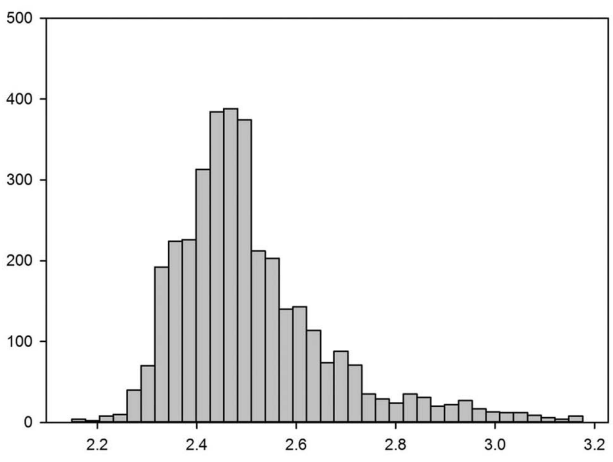

(c)

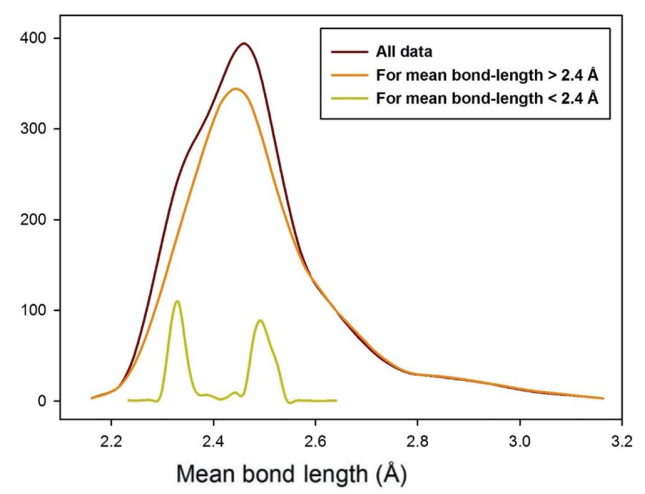

(d)

Figure 12

Mean bond-length distribution of ${ }^{[8]} \mathrm{Ca}^{2+}$ bonded to $\mathrm{O}^{2-}(a)$. Bond-length distribution for ${ }^{[8]} \mathrm{Ca}^{2+}$ bonded to $\mathrm{O}^{2-}$ are shown for configurations with a mean bond length $(b)$ less or equal to $2.44 \AA$ (superimposed with its mean bond-length kernel-density estimation; $n=568$ ), and (c) greater than $2.44 \AA(n=3584)$. The effect of the former on the aggregate bond-length distribution is shown via kernel-density estimation $(d)$. 
$\mathrm{Ca}^{2+}$ and $\mathrm{Ba}^{2+}$ up to [7], compared with $\sim 0.15$ for $\mathrm{Na}^{+}$and $\mathrm{Cs}^{+}$ up to [8].

\subsection{Skewness and kurtosis as a function of coordination number}

Figs. $10(c)$ and $(d)$ summarize the variation of skewness and kurtosis as a function of coordination number for the common alkaline-earth-metal ions, respectively. There is a more-or-less linear decrease in skewness with increasing coordination number. Abnormally low values of skewness (and kurtosis) are obtained for the bond-length distributions of ${ }^{[5]} \mathrm{Mg}^{2+}$ and ${ }^{[6]} \mathrm{Ba}^{2+}$, but are likely due to insufficient data. The slopes of these graphs are generally steeper for the alkaline-earth metals than for the alkali metals, meaning that the progressive 'flattening' of the distributions described for the alkali metals is less developed for the alkaline-earth metals.

There is a systematic decrease in kurtosis with coordination number for the alkaline-earth-metal ions (Fig. 10d), with the same anomalies as for skewness (Fig. 10c). The resemblance of the trends for skewness and kurtosis is striking, as is the case for the alkali-metal ions (Figs. $7 c$ and $d$ ). The values of skewness and kurtosis arising from the bond-length distributions of each family are strongly correlated (alkali metals: $R^{2}=$ 0.75 ; alkaline-earth metals: $R^{2}=0.74$ ).

\section{General discussion of bond-length distributions}

\subsection{Skewness}

As noted in $\S 5$, bond-length distributions are expected to resemble a positively skewed Gaussian distribution, and this shape originates from the variation in Born repulsion and Coulomb attraction as a function of interatomic distance. In particular, as the coordination number of a cation increases, the mean bond-length increases and the slope of the Born repulsion curve decreases as the mean cation-anion distances increase. This makes the potential energy curve more symmetrical about the mean bond length and hence the skewness of the distribution of bond lengths should decrease with increasing coordination number. Inspection of Figs. 7(c) and $10(c)$ shows that this is generally the case for the alkali metals and the alkaline-earth metals.

\subsection{Kurtosis}

As noted above, kurtosis is a measure of the distribution of data between the peak and the tails of the distribution: a high kurtosis indicates that the distribution has a sharper maximum and larger tails, and a low kurtosis indicates that the distribution has a rounder maximum and smaller tails. Fig. 11 shows a kernel-density estimation of the bond-length distributions of coordination numbers [6] to [9] for $\mathrm{Na}^{+}$. This example shows decreases in both kurtosis and skewness from coordination [6] to [9]: kurtosis values are $1.317,0.723,0.461$ and 0.121 , and skewness values $3.246,-0.020,-0.197$ and -0.879 , respectively. Fig. 11 shows that the major contributor to kurtosis is the shape of the maximum of the distribution, rather than the length of the tail, as the tails and the minimum and maximum bond lengths are fairly similar for all coordination numbers, but the flattening of the distribution is very notable with increasing coordination number. Figs. $7(d)$ and $10(d)$ show that kurtosis decreases with increasing coordination number for the alkali metals and the alkaline-earth metals; note that the main deviations from this trend are the same as those for the skewness plots, reinforcing the suggestion that small sample size may be the cause of these deviations.

For low coordination numbers, a change in bond valence corresponds to a relatively small change in bond length because of the steepness of the bond-valence-bond-length curve at short bond lengths. For high coordination numbers, a change in bond valence corresponds to a large change in bond length because of the shallowness of the bond-valence-bondlength curve at long bond lengths. Thus ions with low coordination numbers show a lower range in bond lengths, whereas ions with high coordination numbers show a much greater range in bond lengths (Figs. $7 b$ and $10 b$ ), leading to much flatter distributions (i.e. with lower kurtosis) at higher coordination numbers.

\subsection{Multi-modal distributions}

Several cations show multi-modal distributions of their bond lengths. The most prominent bimodal distribution is for

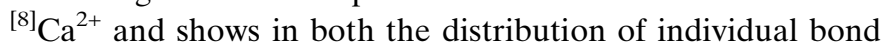

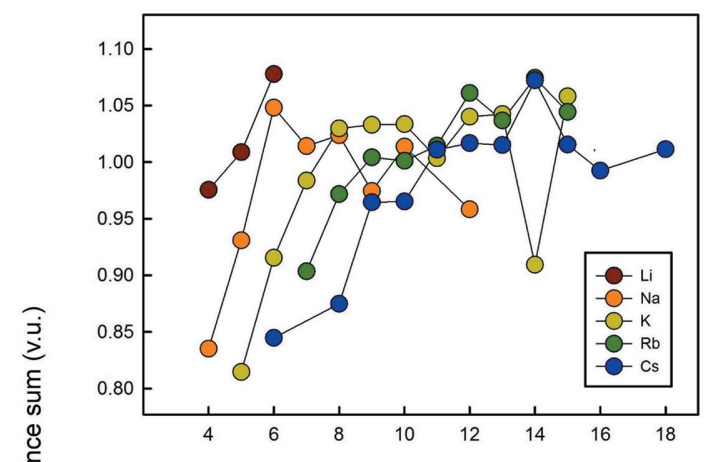

(a)

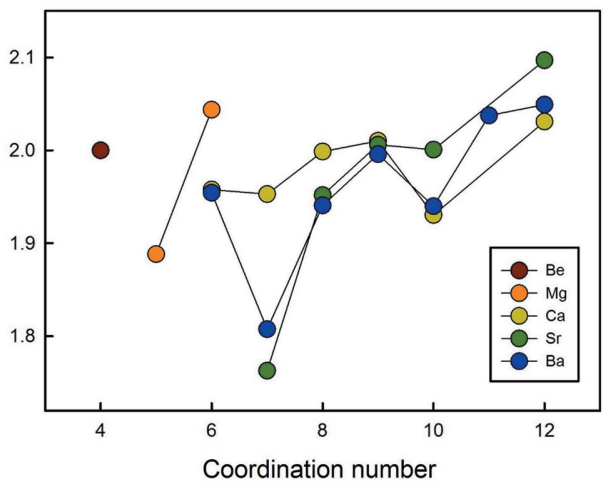

(b)

Figure 13

Bond-valence sum as a function of coordination number for $(a)$ the alkalimetal and $(b)$ alkaline-earth-metal ions using the parameters of Gagné \& Hawthorne (2015). 
Table 3

Bond-valence parameters for large alkali and alkaline-earth metals calculated with and without a hard cut-off of 12 bonds.

\begin{tabular}{llll}
\hline Ion & $R_{\mathrm{o}}(\AA)$ & $B(\AA)$ & RMSD (v.u.) \\
\hline $\mathrm{K}^{+}$ & 2.047 & 0.398 & 0.164 \\
$\mathrm{~K}^{+}(\mathrm{CN} \leq 12)$ & 1.985 & 0.425 & 0.157 \\
$\mathrm{Rb}^{+}$ & 1.993 & 0.478 & 0.150 \\
$\mathrm{Rb}^{+}(\mathrm{CN} \leq 12)$ & 1.780 & 0.577 & 0.148 \\
$\mathrm{Cs}^{+}$ & 2.305 & 0.411 & 0.138 \\
$\mathrm{Cs}^{+}(\mathrm{CN} \leq 12)$ & 1.966 & 0.561 & 0.138 \\
$\mathrm{Ba}^{2+}$ & 2.223 & 0.406 & 0.217 \\
$\mathrm{Ba}^{2+}(\mathrm{CN} \leq 12)$ & 2.208 & 0.417 & 0.215 \\
\hline
\end{tabular}

lengths (Fig. 8f) and mean bond lengths (Fig. 12a). For the distribution of individual bond lengths, there is an intense maximum at $2.49 \AA$ and a less intense maximum at $2.35 \AA$; for the distribution of mean bond lengths, there is an intense maximum at $2.49 \AA$ and a less-intense maximum at $2.42 \AA$. Inspection of the data with $\left\langle{ }^{[8]} \mathrm{Ca}^{2+}-\mathrm{O}^{2-}\right\rangle$ less than $2.44 \AA$ (79 polyhedra) shows that there are 33 garnet structures and 27 vesuvianite structures in this range. The distribution of individual ${ }^{[8]} \mathrm{Ca}^{2+}-\mathrm{O}^{2-}$ distances from polyhedra with a mean bond length of $2.44 \AA$ or less are shown in Fig. 12(b), and the distribution of individual ${ }^{[8]} \mathrm{Ca}^{2+}-\mathrm{O}^{2-}$ distances from polyhedra with a mean bond length greater than $2.44 \AA$ are shown in Fig. 12(c). Fig. 12(b) also shows the kernel density estimation of the mean bond-length distribution of its constituent polyhedra. Removal of the data of Fig. 12(b) from the overall distribution of ${ }^{[8]} \mathrm{Ca}^{2+}-\mathrm{O}^{2-}$ distances gives a single-mode distribution (Fig. 12c). The kernel density estimations for all three distributions are shown in Fig. $12(d)$, and indicate how the bimodal distribution of Fig. 8(f) arises. In the garnet and vesuvianite structures, the bond lengths are split into two equal populations, four larger than the mean and four smaller than the mean. As shown in Fig. 12(d), the population of larger distances merges with the overall distribution of bond lengths and does not materially alter its overall shape, whereas the population of smaller distances lies toward the lower edge of the overall distribution and gives rise to a shoulder on that distribution.

Multi-modal distributions are observed for the following configurations: $\quad{ }^{[10]} \mathrm{Na}^{+}, \quad{ }^{[12]} \mathrm{K}^{+}$, ${ }^{[13]} \mathrm{K}^{+}, \quad{ }^{[14]} \mathrm{K}^{+}, \quad{ }^{[15]} \mathrm{K}^{+}, \quad{ }^{[18]} \mathrm{Cs}^{+}$,

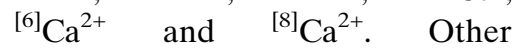
configurations may show deviations from unimodal behaviour, although this is often unclear, possibly due to too few data and/or significant overlap: ${ }^{[4]} \mathrm{Na}^{+},{ }^{[15]} \mathrm{Rb}^{+}$, ${ }^{\left[{ }^{[9]}\right.} \mathrm{Cs}^{+},{ }^{\left[{ }^{10]}\right.} \mathrm{Cs}^{+},{ }^{[12]} \mathrm{Cs}^{+},{ }^{[10]} \mathrm{Ba}^{2+}$. What the example of ${ }^{[8]} \mathrm{Ca}^{2+}$ makes clear is that one must examine the effects of non-random sampling (by large numbers of data on a specific structure type) before ascribing such an effect to any crystal-chemical mechanism.
Mean bond-length distributions for the configurations of the alkali-metal ions bonded to $\mathrm{O}^{2-}$ with a sample size of $100+$ coordination polyhedra: $(a)^{[4]} \mathrm{Li}^{+},(b){ }^{[6]} \mathrm{Li}^{+},(c){ }^{[4]} \mathrm{Na}^{+},(d){ }^{[5]} \mathrm{Na}^{+},(e)^{[6]} \mathrm{Na}^{+},(f)^{[7]} \mathrm{Na}^{+}$, $(g){ }^{[8]} \mathrm{Na}^{+},(h){ }^{[6]} \mathrm{K}^{+},(i){ }^{[8]} \mathrm{K}^{+},(j){ }^{[9]} \mathrm{K}^{+},(k){ }^{[10]} \mathrm{K}^{+},(l){ }^{[12]} \mathrm{K}^{+},(m){ }^{[10]} \mathrm{Rb}^{+}$. 
Table 4

Mean bond-length statistics for the five common alkali-metal ions.

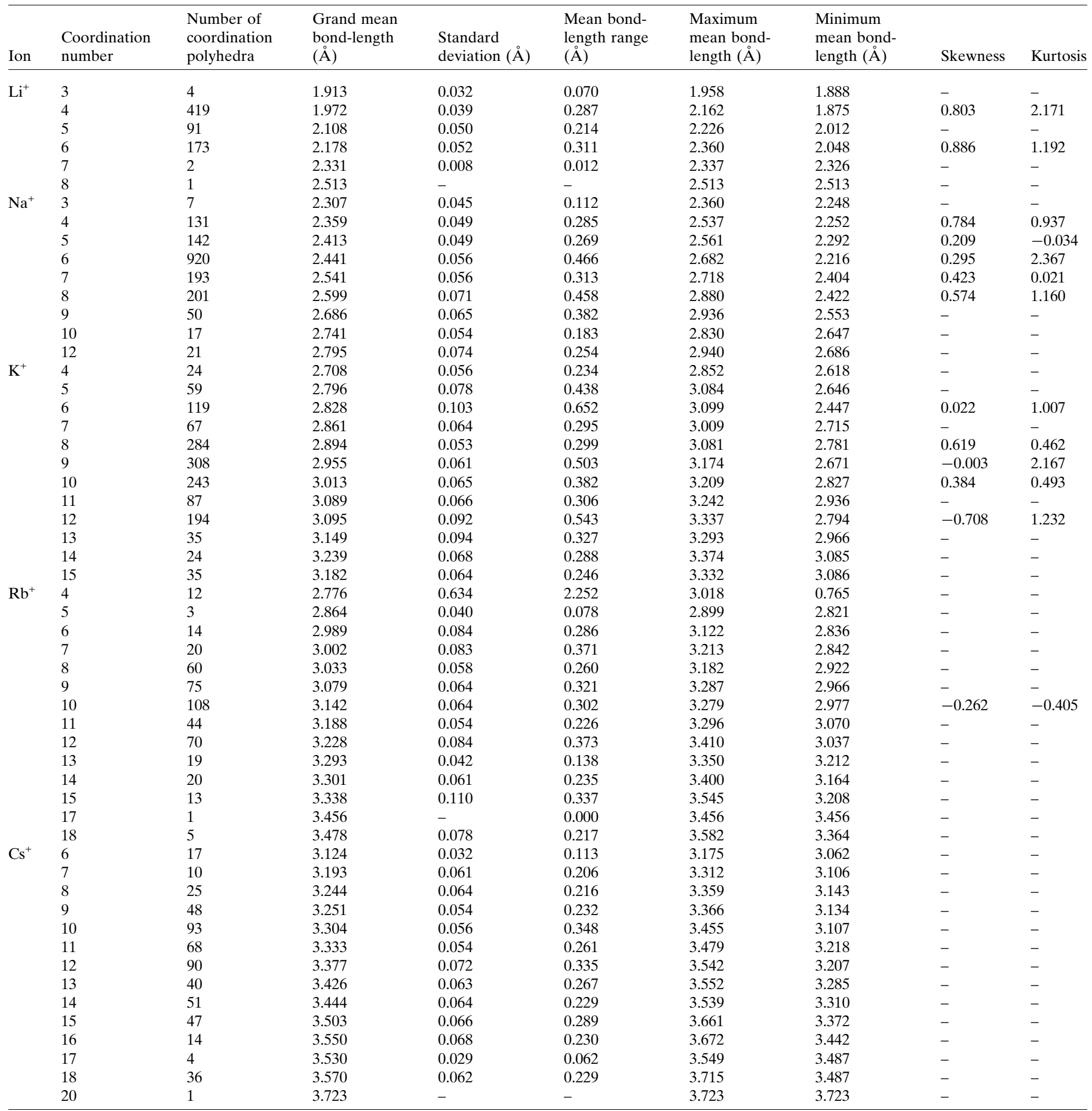

\subsection{Bond valences}

Figs. 13(a) and (b) show the mean bond-valence sum as a function of coordination number, using the parameters of Gagné \& Hawthorne (2015) for those alkali-metal and alkaline-earth metal configurations of 100 bonds or more. There is a general increase in mean bond-valence sum with increasing coordination number. This increase is much larger with smaller coordination numbers and tends to level off at larger
( $>$ [9]) coordination numbers. In the bond-valence model, the incident bond-valence sum for any ion is (approximately) equal to the ion valence, and there should be no correlation between incident bond-valence sum and coordination number of the ion. As indicated in Fig. 13, this is not the case. Moreover, this correlation is much more exaggerated when using the bond-valence parameters of other authors, as Gagné \& Hawthorne (2015) added a coordination-based optimization factor in their method of derivation of bond-valence para- 
meters. However, they were not able to eliminate the correlation between mean bond-valence sum and coordination number for all ions. Correlations of the type shown in Fig. 13 have been noted in the past, but have always been attributed to a limitation in the form of the bond-valence equation used. In particular, it has been suggested (e.g. Wander et al., 2015) that the exponential equation of Brown \& Altermatt (1985) has too shallow a slope at short bond distances and too steep a slope at long bond distances, resulting in short (long) bonds seeming weaker (stronger) than they are. However, this effect is not limited to the equation of Brown \& Altermatt (1985). Gagné \& Hawthorne (2015) tested a series of two- and threeparameter equations, and found that any equation that gives a good fit to the data suffers from the same 'curvature problem' at short and long bond distances. Although the origin of this problem is not clear, this shows that the problem may not lay in the parameterization of the relation, but possibly in a breakdown of the model itself in structures with unusual coordinations. This issue requires further investigating.

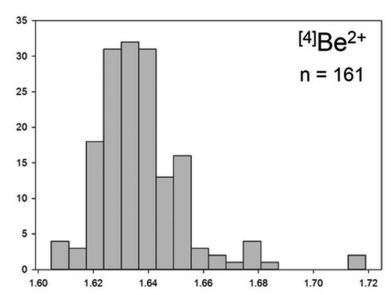

(a)

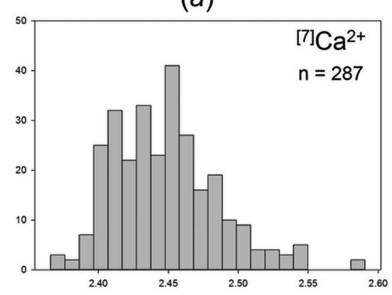

(d)

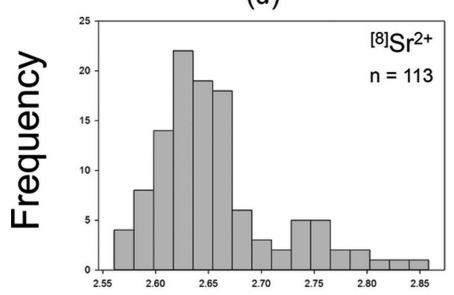

(g)

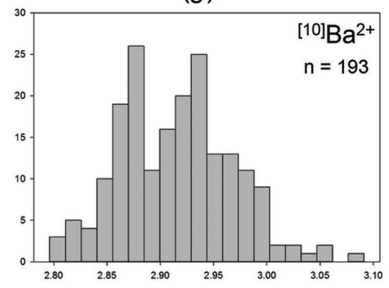

(j)

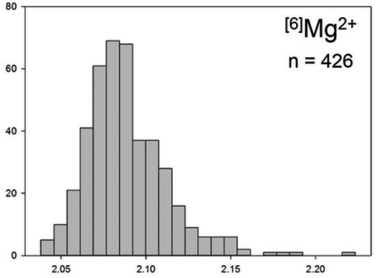

(b)

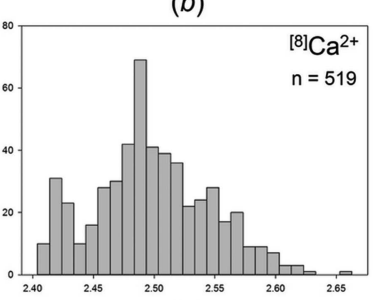

(e)

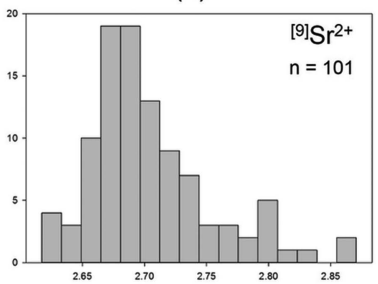

(h)

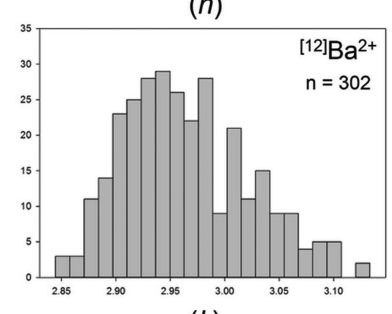

(k)

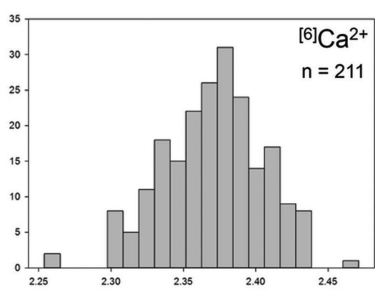

(c)

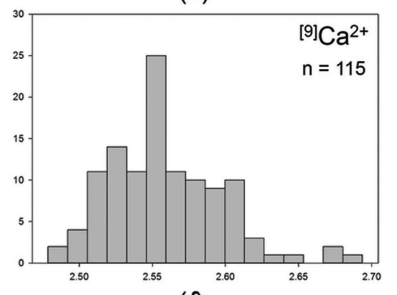

$(f)$

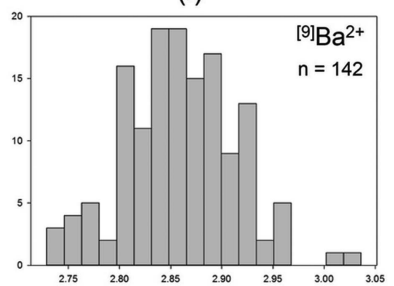

(i)
Gagné \& Hawthorne (2015) give bond-valence parameters for four ions to which they assign coordinations higher than [12]: $\mathrm{K}^{+}, \mathrm{Rb}^{+}, \mathrm{Cs}^{+}$and $\mathrm{Ba}^{2+}$. Table 3 lists their values of the RMSD from the valence-sum rule obtained from their dataset for each ion and calculated using the equation of Brown \& Altermatt (1985)

$$
S_{i j}=\exp \left[\left(R_{\mathrm{o}}-R_{i j}\right) / B\right],
$$

where $R_{i j}$ is the bond length between ions $i$ and $j, S_{i j}$ is the corresponding bond valence, and $R_{\mathrm{o}}$ and $B$ are the bondvalence parameters. Here, we derive new bond-valence parameters using a hard cut-off of 12 bonds for those configurations we observe in coordination numbers greater than [12]. These are also listed in Table 3 with their associated RMSD values. There are minor changes in the $R_{\mathrm{o}}$ parameter for $\mathrm{K}^{+}$ and $\mathrm{Ba}^{2+}$ (2.047 to 1.985 , and 2.223 to $2.208 \AA$, respectively), but there are major differences for $\mathrm{Rb}^{+}$and $\mathrm{Cs}^{+}$(1.993 to 1.780 and 2.305 to $1.966 \AA$, respectively). In all cases, the $B$ parameter increases to offset the decrease in $R_{\mathrm{o}}$ : for $\mathrm{K}^{+}, 0.398$ to $0.425 \AA$; for $\mathrm{Rb}^{+} 0.478$ to $0.577 \AA$; for $\mathrm{Cs}^{+} 0.411$ to $0.561 \AA$; and for $\mathrm{Ba}^{2+} 0.406$ to $0.417 \AA$. It is no surprise that $R_{\mathrm{o}}$ decreases as some of the longer distances are left out, as Gagné \& Hawthorne (2015) showed that $R_{\mathrm{o}}$ is correlated with the mean bond length of an ion (as well as other physical observables such as ionization energy). To compensate for the decrease in $R_{\mathrm{o}}$, the $B$ parameter adjusts to higher values.

We may assess whether or not interatomic distances greater than those used for [12] coordination are valid in the following ways: (1) verify if the mean bond lengths and bond-valence parameters still follow established trends (e.g. with ionization energy) without the longer bonds; (2) verify the valence-sum rule for the bondvalence parameters obtained with and without the cut-off indirectly via the anion bond-valence sums for a set of structures containing these ions; (3) run computational simulations as to whether electron density is observed between the ions at longer distances. Option (3) has no experimental verification and is not considered here.

(1) We plotted mean bond length as a function of $R_{\mathrm{o}}$ for the five

Figure 15

Mean bond-length distributions for the configurations of the alkaline-earth-metal ions bonded to $\mathrm{O}^{2-}$

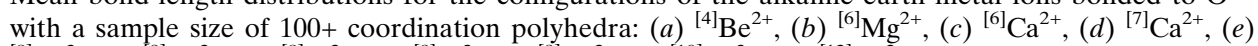
${ }^{[8]} \mathrm{Ca}^{2+},(f){ }^{[9]} \mathrm{Ca}^{2+},(g){ }^{[8]} \mathrm{Sr}^{2+},(h){ }^{[9]} \mathrm{Sr}^{2+},(i){ }^{[9]} \mathrm{Ba}^{2+},(j){ }^{[10]} \mathrm{Ba}^{2+},(k){ }^{[12]} \mathrm{Ba}^{2+}$. 
Table 5

Mean bond-length statistics for the five common alkaline-earth-metal ions.

\begin{tabular}{|c|c|c|c|c|c|c|c|c|c|}
\hline Ion & $\begin{array}{l}\text { Coordination } \\
\text { number }\end{array}$ & $\begin{array}{l}\text { Number of } \\
\text { coordination } \\
\text { polyhedra }\end{array}$ & $\begin{array}{l}\text { Grand mean } \\
\text { bond length } \\
(\AA)\end{array}$ & $\begin{array}{l}\text { Standard } \\
\text { deviation }(\AA)\end{array}$ & $\begin{array}{l}\text { Mean bond- } \\
\text { length range } \\
(\AA)\end{array}$ & $\begin{array}{l}\text { Maximum } \\
\text { mean bond } \\
\text { length }(\AA)\end{array}$ & $\begin{array}{l}\text { Minimum } \\
\text { mean bond } \\
\text { length }(\AA)\end{array}$ & Skewness & Kurtosis \\
\hline \multirow[t]{2}{*}{$\mathrm{Be}^{2+}$} & 3 & 8 & 1.550 & 0.012 & 0.031 & 1.566 & 1.535 & - & - \\
\hline & 4 & 161 & 1.637 & 0.017 & 0.114 & 1.719 & 1.605 & 1.744 & 6.011 \\
\hline \multirow[t]{4}{*}{$\mathrm{Mg}^{2+}$} & 4 & 12 & 1.939 & 0.017 & 0.054 & 1.966 & 1.912 & - & - \\
\hline & 5 & 24 & 1.966 & 0.091 & 0.309 & 2.087 & 1.777 & - & - \\
\hline & 6 & 426 & 2.089 & 0.024 & 0.185 & 2.223 & 2.038 & 1.179 & 3.051 \\
\hline & 8 & 7 & 2.255 & 0.035 & 0.080 & 2.284 & 2.203 & - & - \\
\hline \multirow[t]{7}{*}{$\mathrm{Ca}^{2+}$} & 6 & 211 & 2.371 & 0.034 & 0.217 & 2.471 & 2.254 & -0.261 & 0.236 \\
\hline & 7 & 287 & 2.447 & 0.038 & 0.225 & 2.591 & 2.366 & 0.735 & 0.761 \\
\hline & 8 & 519 & 2.498 & 0.048 & 0.259 & 2.663 & 2.404 & 0.216 & -0.238 \\
\hline & 9 & 115 & 2.559 & 0.039 & 0.215 & 2.694 & 2.479 & 0.761 & 0.927 \\
\hline & 10 & 16 & 2.632 & 0.048 & 0.137 & 2.686 & 2.549 & - & - \\
\hline & 11 & 7 & 2.614 & 0.060 & 0.139 & 2.690 & 2.551 & - & - \\
\hline & 12 & 13 & 2.668 & 0.073 & 0.228 & 2.777 & 2.549 & - & - \\
\hline \multirow[t]{7}{*}{$\mathrm{Sr}^{2+}$} & 6 & 13 & 2.477 & 0.034 & 0.118 & 2.542 & 2.424 & - & - \\
\hline & 7 & 38 & 2.639 & 0.054 & 0.188 & 2.738 & 2.549 & - & - \\
\hline & 8 & 113 & 2.658 & 0.061 & 0.310 & 2.871 & 2.561 & 1.298 & 1.633 \\
\hline & 9 & 101 & 2.703 & 0.051 & 0.266 & 2.871 & 2.604 & 1.119 & 1.523 \\
\hline & 10 & 50 & 2.769 & 0.070 & 0.436 & 3.053 & 2.617 & - & - \\
\hline & 11 & 8 & 2.798 & 0.027 & 0.075 & 2.830 & 2.755 & - & - \\
\hline & 12 & 53 & 2.825 & 0.055 & 0.213 & 2.930 & 2.716 & - & - \\
\hline \multirow[t]{9}{*}{$\mathrm{Ba}^{2+}$} & 6 & 21 & 2.689 & 0.054 & 0.197 & 2.794 & 2.597 & - & - \\
\hline & 7 & 21 & 2.792 & 0.033 & 0.121 & 2.855 & 2.733 & - & - \\
\hline & 8 & 88 & 2.816 & 0.049 & 0.254 & 2.952 & 2.698 & - & - \\
\hline & 9 & 142 & 2.860 & 0.055 & 0.307 & 3.036 & 2.729 & 0.111 & 0.302 \\
\hline & 10 & 193 & 2.915 & 0.053 & 0.295 & 3.091 & 2.796 & 0.282 & -0.022 \\
\hline & 11 & 82 & 2.944 & 0.039 & 0.187 & 3.035 & 2.849 & - & - \\
\hline & 12 & 302 & 2.965 & 0.058 & 0.289 & 3.133 & 2.845 & 0.467 & -0.299 \\
\hline & 13 & 6 & 3.010 & 0.051 & 0.137 & 3.070 & 2.934 & - & - \\
\hline & 14 & 1 & 3.080 & - & - & 3.080 & 3.080 & - & - \\
\hline
\end{tabular}

alkali-metal ions for the values including and excluding bonds with a hard cut-off of [12]. Including the bonds, $R^{2}=0.94$, whereas excluding these bonds, $R^{2}$ drops to 0.79 . Plotting $R_{\mathrm{o}} /$ (mean bond length) against ionization energy (Gagné \& Hawthorne, 2015), $R^{2}$ (inclusive) $=0.35$, whereas $R^{2}$ (exclusive) $=0.01$.

(2) We compared the bond-valence sums of the anions for 19 structures containing $\mathrm{K}^{+}, \mathrm{Rb}^{+}, \mathrm{Cs}^{+}$and $\mathrm{Ba}^{2+}$, which we originally described in coordinations greater than [12], for the following two cases: (1) using the parameters of Gagné \& Hawthorne (2015) and no cut-off, and (2) using new bondvalence parameters (above) that were derived with a cut-off of coordination [12], and doing the evaluation on the 19 structures using a cut-off of [12]. We find that both sets of parameters, used in the way they were derived, give exactly the same result for the anion bond-valence sums (RMSD = 0.128 v.u., or $6.4 \%$ from the nominal oxidation state).

Following (1) and (2) we conclude that the notion of counting bonds up to a maximum of 12 seems unjustified. We can find no strong argument against the occurrence of higher coordination numbers.

\section{Mean bond-length distributions}

Figs. S3 and S4 give all the mean bond-length distributions for the alkali-metal and alkaline-earth-metal ions; those with adequate sample sizes (below) are shown in Figs. 14 and 15. Tables 4 and 5 give the grand mean bond length and standard deviation, the minimum and maximum mean bond length (and range), the skewness and kurtosis of each distribution (where justified by sample size) and the number of coordination polyhedra and coordination numbers for all configurations for the alkali and alkaline-earth metals. A minimum sample size was determined in the same way as above for ${ }^{[6]} \mathrm{Na}^{+}$, less than which the values of skewness and kurtosis have little significance; this threshold was set to 100 coordination polyhedra

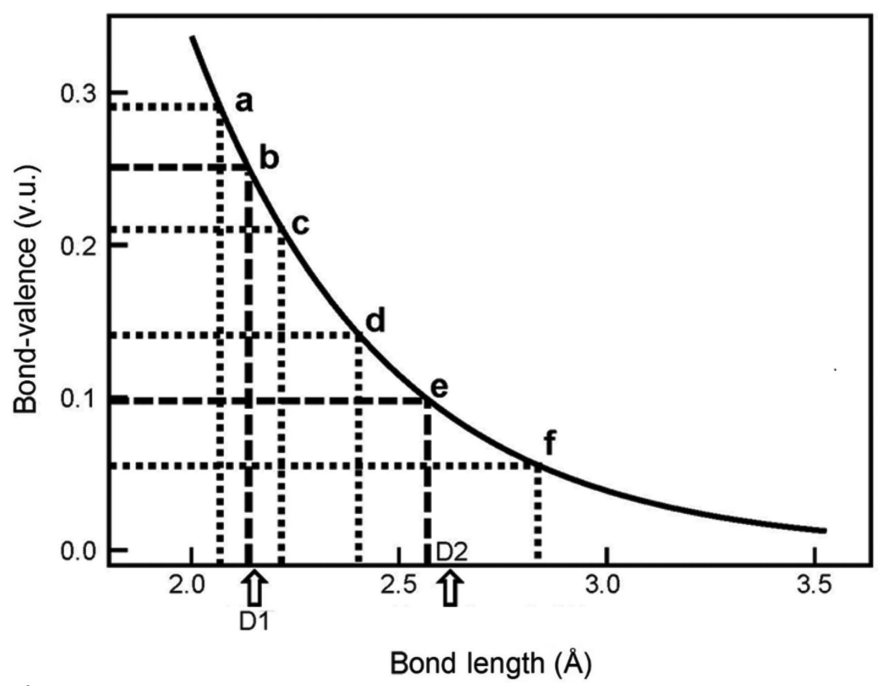

Figure 16

Bond-valence bond-length curve for $\mathrm{Na}^{+}$. The exponential shape leads to the distortion theorem of the bond-valence model. 
and is relatively high due to the wide range of mean bond lengths observed for these families.

The average range of mean bond lengths for the 55 configurations of the alkali metals is $0.308 \AA$, and is $0.217 \AA$ for the 29 configurations of the alkaline-earth metals. The largest range is observed for ${ }^{[6]} \mathrm{K}^{+}$, from 2.447 to $3.099 \AA$. The distribution for ${ }^{[4]} \mathrm{Be}^{2+}$ (Fig. 15a) shows a feature that is of importance in examining bond-length distributions: there is a notable outlier with two $\left\langle{ }^{[4]} \mathrm{Be}^{2+}-\mathrm{O}\right\rangle$ distances at $\sim 1.717 \AA$. These values are for sørensenite, ideally $\mathrm{Na}_{4} \mathrm{Sn}^{4+}\left[\mathrm{Be}_{2-}\right.$ $\left.\mathrm{Si}_{6} \mathrm{O}_{18}\right]\left(\mathrm{H}_{2} \mathrm{O}\right)_{2}$ (Metcalf-Johansen \& Hazell, 1976), which was assumed to have the ideal composition in the structure study. However, inspection of the chemical analyses listed by Semenov et al. (1965) shows that the formulae depart significantly from the ideal stoichiometry used to interpret the structure results. In many pegmatite minerals, $\mathrm{Be}^{2+}$ is commonly partly substituted by $\mathrm{Li}^{+}$or $\mathrm{Al}^{3+}$, both of which are larger than $\mathrm{Be}^{2+}$ (Shannon, 1976) and this may be what has happened here. Of course, this is speculation, but emphasizes the importance of electron- and ion-microprobe analysis of the specific crystal used to collect X-ray intensity data for structure analysis of minerals.

\subsection{The effect of distortion}

10.1.1. The distortion theorem. The distortion theorem states that for any ion, lengthening some bonds and shortening others, keeping the bond-valence sum the same, will always increase the mean bond length due to the exponential nature of the relation (Brown, 2002). Here we use the following definition of bond-length distortion from the mean value in a polyhedron

$$
\Delta=\frac{1}{n} \sum_{i=1}^{n}\left[\frac{\left(R_{i}-\bar{R}\right)}{\bar{R}}\right]^{2},
$$

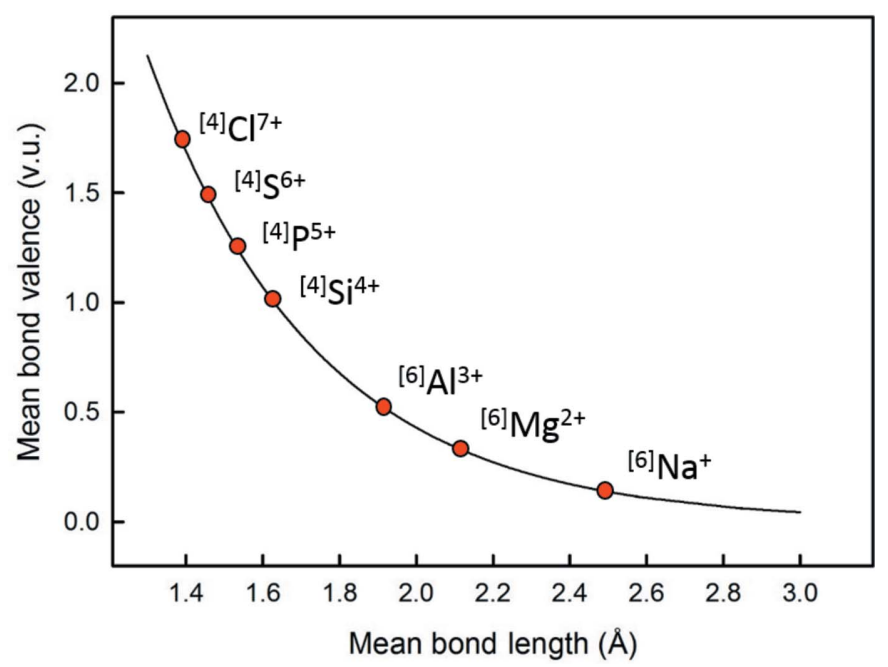

Figure 17

Bond-valence curve for the $\mathrm{Na}$ isoelectronic series, and ideal mean bondvalence and associated mean bond-length for the most common coordination number of each ion of the series. where $R_{i}$ is the length of bond $i, \bar{R}$ is the mean bond length and the summation is taken over the $n$ bonds of the polyhedron.

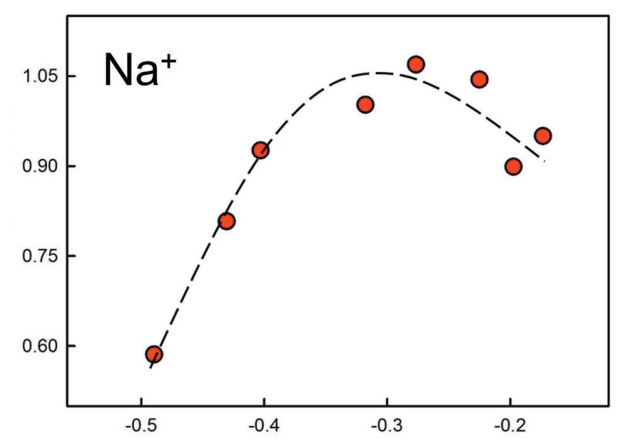

(a)

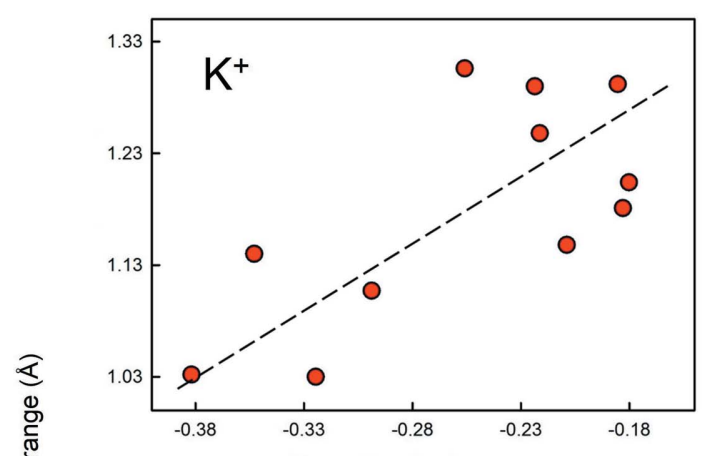

(b)

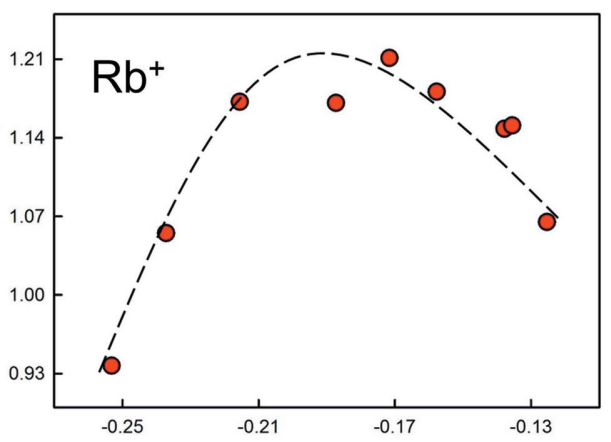

(c)

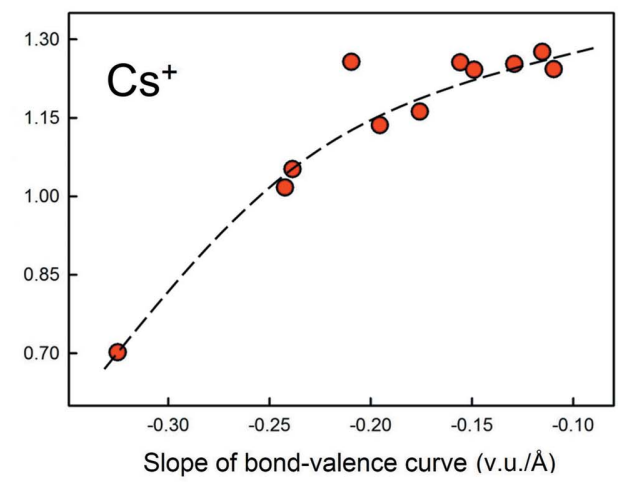

(d)

Figure 18

Variation in the observed bond-length ranges for the different coordination numbers of $(a) \mathrm{Na}^{+},(b) \mathrm{K}^{+},(c) \mathrm{Rb}^{+}$and $(d) \mathrm{Cs}^{+}$bonded to $\mathrm{O}^{2-}$ as a function of the slope of the bond-valence curve at the mean bond-length corresponding to those coordinations. The dashed lines are drawn as a guide to the eye (they are not least-squares fits to the data). 
This expression was designated the quadratic elongation by Robinson et al. (1971) and distortion by Brown \& Shannon (1973), and was used by many previous authors as a measure of distortion as it is the standard deviation of the mean bond length in a specific polyhedron, i.e. the measure of dispersion of the individual bond lengths. The distortion is shown graphically in Fig. 16 with the bond-valence curve for $\mathrm{Na}^{+}$. For [4]-coordinated $\mathrm{Na}^{+}$, the mean bond valence is 0.25 v.u., marked by the dashed line intersecting the bond-valence curve at point $b$ (Fig. 16). If half the bonds shorten and half the bonds lengthen to $a$ and $c$, respectively, on the bond-valence curve, the mean bond length (marked by D1 in Fig. 16) increases slightly over the mean bond length for four equal bonds. For [10]-coordinated Na, the mean bond valence is 0.10 v.u., marked by the dashed line intersecting the bondvalence curve at point $e$ (Fig. 16). If half the bonds shorten and half the bonds lengthen to $d$ and $f$, respectively, on the bondvalence curve, the mean bond length (marked by D2 in Fig. 16) increases considerably more over the mean bond length for ten equal bonds.

Secondly, Fig. 16 shows that the effect of the distortion theorem is greatly affected by the slope and curvature of the bond-valence-bond-length relation; if we use the same concept for points $a, b$ and $c$, we see that the mean bond length changes very little, and much less relative to points $d, e$ and $f$. Different ions have their mean bond length at different points (curvature) on this graph, and thus are affected differently by the distortion theorem. The best way to visualize this concept is by making use of a universal curve as described in Brown \& Shannon (1973), where the relation for an isoelectronic series of ions can be described by the same bond-valence curve (with a slight decrease in fit compared with ion-based curves). In Fig. 17 we show the bond-valence curve for the $\mathrm{Na}$ isoelectronic series $\left(R_{\mathrm{o}}=1.630, B=0.438\right)$, and identify the ideal mean bond-valence and associated mean bond length for the most common coordination number of each ion of the series. We see that ions of lower charge, and with generally higher coordination numbers, occur on part of the bond-valence curve that is much more susceptible to higher bond-length distortion (i.e. with lower bond valences). Hence, the alkali metals are more strongly affected by distortion than the alkaline-earth metals, and so on. Figs. 18(a) $-(d)$ show the variation in the range of bond lengths for different coordination numbers as a function of the slope of the bond-valence curve at the mean bond length corresponding to those coordination numbers for $\mathrm{Na}^{+}, \mathrm{K}^{+}, \mathrm{Rb}^{+}$and $\mathrm{Cs}^{+}$. As predicted above, there is a positive correlation between range and slope for each cation; the trends are well developed for $\mathrm{Na}^{+}, \mathrm{Rb}^{+}$ and $\mathrm{Cs}^{+}$, despite a decrease at the highest coordination number for $\mathrm{Na}^{+}$and $\mathrm{Rb}^{+}$possibly due to fewer data, but is perturbed by considerable scatter for $\mathrm{K}^{+}$. As noted above, the distortion will also be correlated with the curvature (as well as the slope) of the bond-valence curve, but the slope and curvature are highly correlated and their effect is well represented by just the slope of the curve.

10.1.2. Grand mean bond length. Using the bond-valence parameters of Gagné \& Hawthorne (2015), we calculated the predicted grand mean bond length for all configurations of the alkali-metal and alkaline-earth-metal ions by converting the mean bond-valence (i.e. the Pauling bond strength) of the coordination polyhedron into a mean bond length, where all bonds of the idealized polyhedron have the same length. When comparing the observed grand mean bond length (Tables 4 and 5) to the calculated values, we obtain an overall difference of $2.5 \%$ for the alkali metals $(1.2 \%$ when weighted by the number of coordination polyhedra), and $1.0 \%(0.8 \%$ weighted) for the alkaline-earth metals. As shown in Fig. 19, the predicted values closely follow the observed values, but are slightly larger and the difference increases with increasing mean bond length, in accordance with the idea that distortion of coordination polyhedra causes an increase in mean bond length, and that this effect should increase with increasing coordination number.

For any ion configuration we may calculate the maximum amount of distortion that is compatible with its observed bond-length distribution, using the bond-valence model and the minimum and maximum bond length observed for that configuration. We have done this for all configurations of the alkali-metal and alkaline-earth-metal ions, by using the minimum and maximum bond length observed for each configuration and distributing the other bond lengths in a way that maximizes distortion while satisfying the valence-sum rule. This procedure resulted in a mean potential distortion of $3.9 \%$ for the 55 alkali-metal configurations $(4.6 \%$ when weighted by the number of coordination polyhedra), compared with $2.5 \%$ ( $1.2 \%$ weighted) for the observed values, and $2.8 \%$ (3.4\% weighted) for the 29 alkaline-earth-metal configurations, compared with $1.0 \%(0.8 \%$ weighted) for the observed values. These calculations show that for most ion configurations, the observed ranges of bond lengths are significantly larger than is compatible with the observed

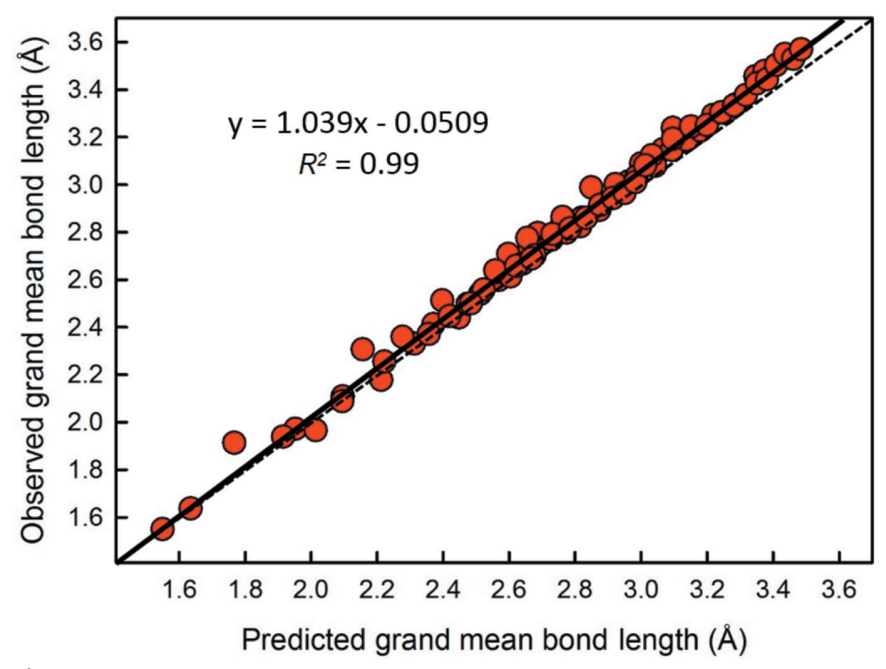

Figure 19

Observed versus predicted values of the grand mean bond length for all observed ion configurations of the alkali-metal and alkaline-earth-metal ions. The observed values of mean bond length are usually larger than the values predicted by the bond-valence curve for equidistant bonds. The dashed line is for $y=x$. 
distortions. Thus, in addition to distortion, other factors must also affect mean bond lengths.

10.1.3. Mean bond length as a function of distortion. Fig. 20 (a) shows the mean bond length for ${ }^{[6]} \mathrm{Na}^{+}-\mathrm{O}^{2-}$ as a function of distortion $(n=920)$. There is a positive correlation between mean bond length and distortion, $\Delta$, for reasons discussed above; the inclined solid line shows the result of a linear regression $\left(R^{2}=0.263\right)$. The calculated curve for the effect that distortion has on mean bond length for ${ }^{[6]} \mathrm{Na}^{+}-\mathrm{O}^{2-}$ is shown by the dashed line on Fig. 20(a). The difference between observed and predicted curve is partly due to the data near $\Delta=0$ (discussed below) which has a very large

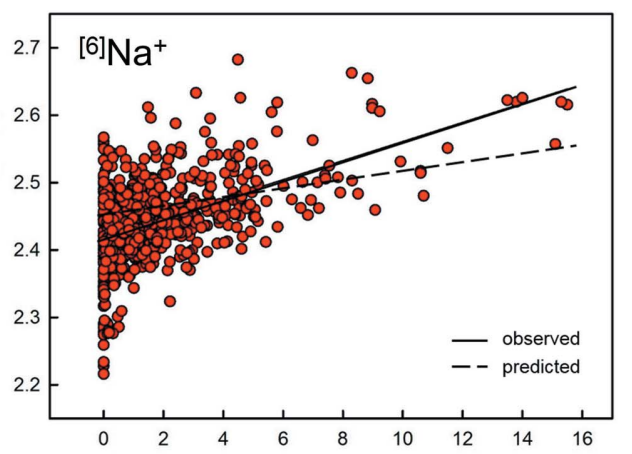

(a)

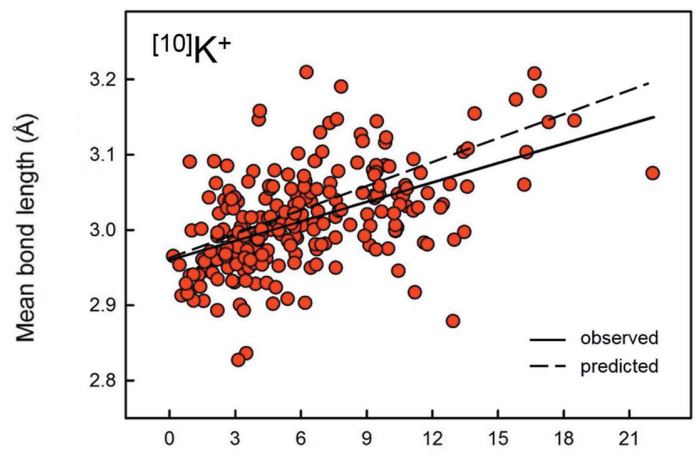

(b)

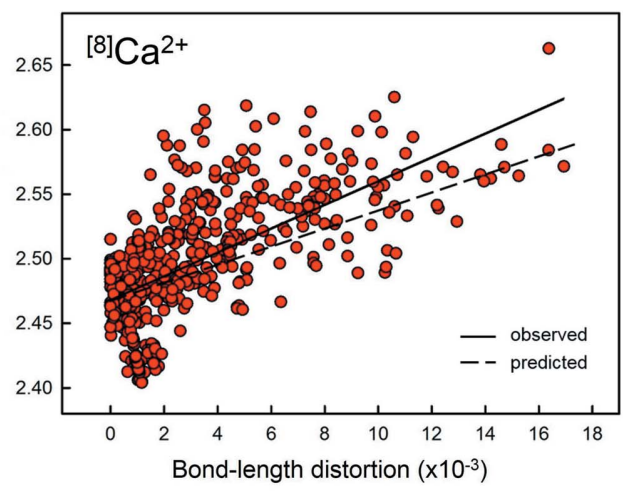

(c)

Figure 20

The effect of bond-length distortion on mean bond length for $(a)^{[6]} \mathrm{Na}^{+}$, (b) ${ }^{[10]} \mathrm{K}^{+}$and $(c){ }^{[8]} \mathrm{Ca}^{2+}$. The positive correlation indicates that distortion of the coordination polyhedron has a considerable effect on the mean bond length of the constituent polyhedron. The dashed line indicates the predicted effect of distortion on mean bond length for that ion configuration according to the bond-valence curve of the ion. scatter. Additionally, we note that highly distorted configurations generally have poor agreement with the valence-sum rule, which leads to variability in the data above and below the predicted curve. However, highly distorted polyhedra for

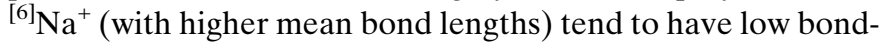
valence sums $(\sim 0.8-0.9$ v.u. $)$, indicating that the mean bond lengths of these polyhedra are larger than predicted from the valence-sum rule and the form of the bond-valence curves.

A very prominent feature of Fig. 20(a) is the fact that the widest range of mean bond lengths occurs at zero distortion; $\left\langle{ }^{[6]} \mathrm{Na}^{+}-\mathrm{O}^{2-}\right\rangle$ varies from 2.216 to $2.567 \AA$ at $\Delta=0$ alone, compared with 2.276 to $2.682 \AA$ for all other data with $\Delta \neq 0$, and the range of observed mean bond lengths decreases as $\Delta$ increases. The total range in mean bond length is $0.466 \AA$; this may be compared with the predicted range that may be assigned to the effect of distortion (dashed line), $0.11 \AA$, as well as the observed range (solid line), $0.21 \AA$ (Fig. 20a).

We give the analogous bond-length distortion plots for the 55 configurations of the alkali-metal ions and 29 configurations of the alkaline-earth-metal ions in Figs. S5 and S6, respectively. We note that this concentration of data at $\Delta=0$ is evident for $\mathrm{Li}^{+}$in [4]- and [6]-coordination, $\mathrm{Na}^{+}$in [4]- and [6]coordination and $\mathrm{K}^{+}$in [6]-coordination, and occurs more subtly for $\mathrm{Na}^{+}$in [5]- and [8]-coordination as well as $\mathrm{K}^{+}$in [8][9] and [12]-coordination. For the alkaline-earth metals, this concentration of data at $\Delta=0$ occurs for $\mathrm{Be}^{2+}$ [4]-coordinated, $\mathrm{Mg}^{2+}$ [4]- and [6]-coordinated, $\mathrm{Ca}^{2+}[6]-$ and [12]-coordinated, $\mathrm{Sr}^{2+}$ [6]-coordinated and $\mathrm{Ba}^{2+}$ [12]-coordinated, and more subtly for $\mathrm{Ca}^{2+}[7]-$ and [8]-coordinated, $\mathrm{Sr}^{2+}[8]$-coordinated and $\mathrm{Ba}^{2+}[8]$ and [10]-coordinated. In Fig. 20(b), we show the distortion plot of ${ }^{[10]} \mathrm{K}^{+}$, which in contrast to that for ${ }^{[6]} \mathrm{Na}^{+}$ (Fig. 20a) shows no preference for $\Delta=0$. It is therefore interesting to see that the observed mean bond-length values (solid line) are generally lower than what we predict over the whole range of distortion (dashed line) because of the absence

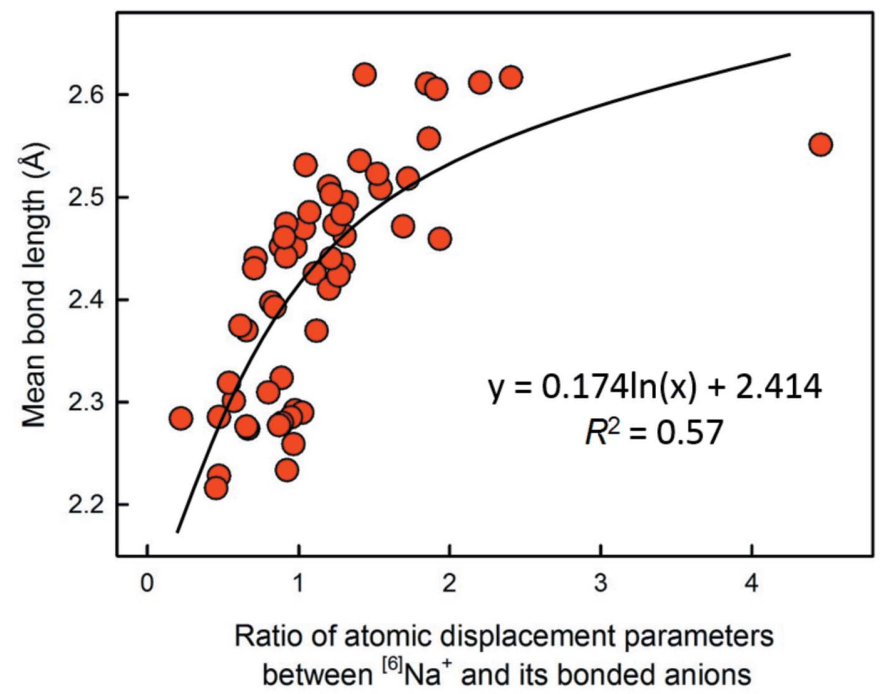

Figure 21

The correlation of atomic displacement with mean bond length. Values are for the ratio of the atomic displacement parameters between ${ }^{[6]} \mathrm{Na}^{+}$ and its bonded anions $(n=56)$. 
of the large scatter and amount of data near $\Delta=0$ that greatly affects the slope of the observed curve for ion configurations with significant scatter at $\Delta=0$. Fig. $20(c)$ shows ${ }^{[8]} \mathrm{Ca}^{2+}$ as an intermediate configuration with some concentration of data near $\Delta=0$. Some of these differences may be due to the distortion parameter that we use not representing different distributions of bond lengths within a polyhedron, as this does affect somewhat the behaviour of mean bond length as a function of distortion (Urusov, 2003, 2014). However, this issue does not affect the mean bond length at zero distortion. Thus it is apparent from the large amount of scatter in Fig. 20 (Figs. S5 and S6) that much of the variation in mean bond length shown in Figs. 14 and 15 (Figs. S3 and S4) is not due to distortion, and that one or more other factors must also affect mean bond length.

\subsection{Atomic displacement}

We selected a sample of 56 coordination polyhedra from the parent distribution of ${ }^{[6]} \mathrm{Na}^{+}$(920 coordination polyhedra) to examine other possible factors that may correlate with mean bond length; these samples were selected so that they were representative of all values of distortion.

When examining the data for outliers that were possible erroneous data, we noticed very large relative variations in anisotropic displacement or equivalent isotropic displacement parameters in the atoms of the parent structures. Cursory examination indicated that the magnitudes of these meanbond-length outliers often correlated with the atomic displacement of the constituent cations and/or anions, suggesting that the central cation responds to an overly large coordination by increasing its dynamic (or static) displacement, while anions respond to an overly small cation-coordination environment by increasing their own displacement. Shannon (1993) and Shannon \& Rossman (1992) have commented on the effect of the former when considering the additivity of fictive dielectric constants of ions in crystals, denoting this behaviour as 'rattling'. Inspection of many structures eventually showed that the ratio $U_{\text {eq(Na) }} / U_{\text {eq(bonded anions) }}$ was most highly correlated with variation in mean bond length. This is shown in Fig. 21 ; the observed correlation is logarithmic, with $R^{2}=0.57$. The fit of the correlation is of the same magnitude as that observed for distortion for the same sample of 56 coordination polyhedra $\left(R^{2}=0.52\right)$. We note that Fig. 21 contains an apparent outlier with an atomic displacement ratio of 4.46 , and that this single data point has considerable effect on whether the shape of the regression curve is logarithmic or linear. Removing this data point, a linear regression gives $R^{2}=0.62$, while the logarithmic fit becomes $R^{2}=0.58$. However, we found no justification for the removal of this data point upon examination of the structure.

10.3. The relation between mean bond length and atomic displacement

The correlation of mean bond length with the atomic displacement parameter has not been discussed extensively in previous work on variation in mean bond lengths. In well ordered crystal structures, there is generally a positive correlation between atomic displacement parameters and coordination number. As shown above, for ${ }^{[6]} \mathrm{Na}^{+}$there is a positive correlation between atomic displacement parameters and mean bond length. Taken together, these two observations suggest that the atomic displacements increase as the strength of the constituent chemical bonds decreases, and that such variation in atomic displacement accompanies variation in bond lengths that occur due to other factors such as bondlength distortion.

For a specific cation with a specific coordination number, one expects the following sequence: (1) over a particular range of distances, the atomic displacement increases with increasing distance; (2) with further increase in distance, continuous displacement changes to discontinuous displacement, i.e. hopping of the central cation in an overly large coordination polyhedron; (3) static displacement of the cation away from the centre of the coordination polyhedron; (4) collapse of the anions forming the coordination polyhedron (perhaps via a ferroelastic phase transition), reducing the coordination number and changing the symmetry of the structure. When considering the factors affecting bond length, we need to recognize the relation between the type of displacement behaviour of the central cation and mean bond-length. In stage (1), there is an increase in mean bond length due to local differences in structures (together with a monotonic change in vibrational displacement). In stage (2), the question arises as to whether the observed mean distances are comparable with those of stage (1) as they are accompanied by large displacement parameters characteristic of atom hopping. In stage (3) the observed distances are not affected by atom hopping, but a change in coordination number of the central cation may be observed. When examining variation in mean bond length for a specific ion in a particular coordination, it is important to limit the data to structures at stage (1), as once hopping occurs, an additional component is added to the measured mean bond length that is not present at smaller distances.

\section{Summary}

(1) We have examined the bond-length distributions for 55 configurations of alkali-metal ions and 29 configurations of alkaline-earth-metal ions, for 4859 coordination polyhedra and 38594 bond distances (alkali metals) and for 3038 coordination polyhedra and 24487 bond distances (alkaline-earth metals).

(2) Bond lengths generally show a positively skewed Gaussian distribution that originates from the variation in Born repulsion and Coulomb attraction as a function of interatomic distance.

(3) The skewness and kurtosis of these distributions generally decrease with increasing coordination number of the central cation, a result of decreasing Born repulsion with increasing coordination number.

(4) We confirm the following minimum coordination numbers: ${ }^{[3]} \mathrm{Li}^{+},{ }^{[3]} \mathrm{Na}^{+},{ }^{[4]} \mathrm{K}^{+},{ }^{[4]} \mathrm{Rb}^{+},{ }^{[6]} \mathrm{Cs}^{+},{ }^{[3]} \mathrm{Be}^{2+},{ }^{[4]} \mathrm{Mg}^{2+}$, ${ }^{[6]} \mathrm{Ca}^{2+},{ }^{[6]} \mathrm{Sr}^{2+}$ and ${ }^{[6]} \mathrm{Ba}^{2+}$, but note that some reported 
examples are the result of extensive dynamic and/or positional short-range disorder and are not ordered arrangements.

(5) Some distributions of bond lengths are distinctly multimodal (primarily bimodal), but for the alkali-metal and alkaline-earth-metal ions, this is often due to the occurrence of large numbers of structure refinements of a particular structure-type in which a particular cation is always present, e.g. for ${ }^{\left[{ }^{[8]}\right.} \mathrm{Ca}^{2+}$, in which many refinements of garnet and vesuvianite structures lead to an over-representation of specific bond lengths.

(6) For alkali-metal and alkaline-earth-metal ions, there is a positive correlation between incident bond-valence sum at the central cation and coordination number, the values varying from 0.84 v.u. for ${ }^{[5]} \mathrm{K}^{+}$to 1.06 v.u. for ${ }^{[8]} \mathrm{Li}^{+}$, and from 1.76 v.u. for ${ }^{[7]} \mathrm{Ba}^{2+}$ to 2.10 v.u. for ${ }^{[12]} \mathrm{Sr}^{2+}$.

(7) Unusually small or large coordination numbers are commonly associated with anomalous values of atomic displacement of the constituent cations and/or anions.

(8) For a sample of ${ }^{[6]} \mathrm{Na}$, the ratio $U_{\text {eq(Na) }} / U_{\text {eq(bonded anions) }}$ is partially correlated with $\left\langle{ }^{[6]} \mathrm{Na}-\mathrm{O}\right\rangle$ mean bond length $\left(R^{2}=\right.$ $0.57)$, suggesting that the vibrational/displacement characteristics of the constituent ions are affected by mean bond length for a fixed coordination number.

(9) Mean bond lengths show a weak correlation with bondlength distortion from the mean value, but clearly also correlate with one or more other factors, e.g. atomic displacement. In particular, some coordination numbers show the widest variation in mean bond length for zero distortion, e.g. $\mathrm{Li}^{+}$in [4]- and [6]-coordination, $\mathrm{Na}^{+}$in [4]- and [6]-coordination and $\mathrm{K}^{+}$in [6]-coordination, and for [4]-coordinated $\mathrm{Be}^{2+}$, [4]- and [6]-coordinated $\mathrm{Mg}^{2+}$, [6]- and [12]-coordinated $\mathrm{Ca}^{2+}$, [6]-coordinated $\mathrm{Sr}^{2+}$ and [12]-coordinated $\mathrm{Ba}^{2+}$.

(10) Bond-valence parameters for the four ions observed in coordinations higher than [12], $\mathrm{K}^{+}, \mathrm{Rb}^{+}, \mathrm{Cs}^{+}$and $\mathrm{Ba}^{2+}$ (Gagné \& Hawthorne, 2015) were calculated for a maximum coordination number of [12]. Both sets of parameters give exactly the same result for anion bond-valence sums. However, the bond-valence parameters calculated for a maximum coordination number of [12] show much poorer correlation with mean observed bond length and no correlation at all with ionization energy of the central cation, in contrast to the bondvalence parameters of Gagné \& Hawthorne (2015).

\section{Acknowledgements}

This work was funded by UM Duff Roblin and GETS Fellowships, and an NSERC PGS-D3 Scholarship to OCG, and a Canada Research Chair and a Discovery grant to FCH from the Natural Sciences and Engineering Research Council of Canada, and by Canada Foundation for Innovation grants to $\mathrm{FCH}$.

\section{References}

Baur, W. H. (1971). Am. Mineral. 56, 1573-1599.

Brown, I. D. (2002). The Chemical Bond in Inorganic Chemistry. Oxford University Press.

Brown, I. D. \& Altermatt, D. (1985). Acta Cryst. B41, 244-247.

Brown, I. D. \& Shannon, R. D. (1973). Acta Cryst. A29, 266-282.
Burns, P. C., Ewing, R. C. \& Hawthorne, F. C. (1997). Can. Mineral. 35, 1551-1570.

Chaminade, J.-P., Gravereau, P., Jubera, V. \& Fouassier, C. (1999). J. Solid State Chem. 146, 189-196.

Chenevas, J., Joubert, J. C. \& Marezio, M. (1975). J. Solid State Chem. 14, 25-32.

Fallon, G. D. \& Gatehouse, B. M. (1980). J. Solid State Chem. 34, $193-$ 198.

Gagné, O. C. \& Hawthorne, F. C. (2015). Acta Cryst. B71, 562-578.

Gatehouse, B. M. \& Grey, I. E. (1983). J. Solid State Chem. 46, 151155.

Haddad, A. \& Jouini, T. (1997). J. Solid State Chem. 134, 10-16.

Hawthorne, F. C. \& Huminicki, D. M. C. (2002). Rev. Mineral. Geochem. 50, 333-403.

Hawthorne, F. C., Krivovichev, S. V. \& Burns, P. C. (2000). Rev. Mineral. Geochem. 40, 1-112.

Horiuchi, H., Saito, A., Tachi, T. \& Nagasawa, H. (1997). Am. Mineral. 82, 143-148.

Huminicki, D. M. C. \& Hawthorne, F. C. (2002). Rev. Mineral. Geochem. 48, 123-253.

Iyi, N., Göbbels, M. \& Matsui, Y. (1995). J. Solid State Chem. 120, 364-371.

Jahn, H. A. \& Teller, E. (1937). Proc. R. Soc. A, 161, 220-235.

Jubera, V., Gravereau, P., Chaminade, J. P. \& Fouassier, C. (2001). J. Solid State Chem. 156, 161-167.

Kahlenburg, V. (2002). Solid State Sci. 4, 183-189.

Lenaz, D., Skogby, H., Princivalle, F. \& Halenius, U. (2004). Phys. Chem. Miner. 31, 633-642.

Leoni, S., Niewa, R., Akselrud, L., Prots, Y., Schnelle, W., Göksuc, T., Cetinkol, M., Somer, M. \& Kniep, R. (2005). Z. Anorg. Allg. Chem. 631, 1818-1824.

Lii, K. H., Wang, Y. P., Chneg, C. Y., Wang, S. L. \& Ku, H. C. (1990). J. Chin. Chem. Soc. 37, 141-149.

Lipp, C. \& Schleid, T. (2006). Z. Anorg. Allg. Chem. 632, 2226-2231.

Majzlan, J., Drahota, P. \& Filippi, M. (2014). Rev. Mineral. Geochem. 79, 17-184.

Metcalf-Johansen, J. \& Hazell, R. G. (1976). Acta Cryst. B32, $2553-$ 2556.

Mills, S. J. \& Christy, A. G. (2013). Acta Cryst. B69, 145-149.

Möller, A. (1998). Chem. Mater. 10, 3196-3201.

Ozaki, Y., Ghedira, M., Chenavas, J., Joubert, J. C. \& Marezio, M. (1977). Acta Cryst. B33, 3615-3617.

Pushcharovskii, D. Y., Gobetchia, E. R., Pasero, M., Merlino, S. \& Dimitrova, O. V. (2002). J. Alloys Compd. 339, 70-75.

Richardson, I. G. (2013). Acta Cryst. B69, 150-162.

Robinson, K., Gibbs, G. V. \& Ribbe, P. H. (1971). Science, 172, 567570.

Sabrowsky, H., Mertens, P. \& Thimm, A. (1985). Z. Kristallogr. 171, 16.

Sabrowsky, H. \& Schroeer, U. (1982). Z. Naturforsch. B. Anorg. Chem. Org. Chem. 37, 818-819.

Sabrowsky, H., Thimm, A. \& Vogt-Mertens, P. (1985). Z. Naturforsch. $B, \mathbf{4 0}, 1761-1762$.

Sabrowsky, H. \& Vogt, P. (1987). Z. Anorg. Allg. Chem. 553, 226230.

Schindler, M., Hawthorne, F. C. \& Baur, W. H. (2000). Chem. Mater. 12, 1248-1259.

Semenov, E. I., Gerassimovsky, V. I., Maksimova, N. V., Andersen, S. \& Petersen, O. V. (1965). Meddelelser om Grønland, 181, 1-19.

Shannon, R. D. (1976). Acta Cryst. A32, 751-767.

Shannon, R. D. (1993). J. Appl. Phys. 73, 348-366.

Shannon, R. D. \& Rossman, G. R. (1992). Am. Mineral. 77, 94-100.

Urusov, V. S. (2003). Z. Kristallogr. 218, 709-718.

Urusov, V. S. (2014). J. Struct. Chem. 55, 1277-1292.

Wander, C. F. M., Bickmore, B. R., Lind, L., Andros, C., Hunt, J., Checketts, H. \& Goodell, T. (2015). Am. Mineral. 100, 160-171.

Wilkens, J. \& Müller-Buschbaum, Hk. (1991). J. Alloys Compd, 177, L31-L33. 